Article

\title{
Condorcet Completion Methods that Inhibit Manipulation through Exploiting Knowledge of Electorate Preferences
}

\author{
Richard F. Potthoff
}

Department of Political Science and Social Science Research Institute, Duke University, Erwin Mill, Duke Box 90420, Durham, NC 27708, USA; E-Mail: potthoff@duke.edu; Tel.: +1-919-684-1380

External Editor: Ulrich Berger

Received: 4 August 2014; in revised form: 2 October 2014 / Accepted: 6 October 2014 / Published: 30 October 2014

\begin{abstract}
This paper attacks a problem like the one addressed in an earlier work (Potthoff, 2013) but is more mathematical. The setting is one where an election is to choose a single winner from $m(>2)$ candidates, it is postulated that voters have knowledge of the preference profile of the electorate, and preference cycles are limited. Both papers devise voting systems whose two key goals are to select a Condorcet winner (if one exists) and to resist manipulation. These systems entail equilibrium strategies where everyone votes sincerely, no group of voters sharing the same preference ordering can gain by deviating given that no one else deviates, and the Condorcet candidate wins. The present paper uses two unusual ballot types. One asks voters to rank the candidates with respect both to their own preferences and to their discerned order of preference of the entire electorate. The other just asks voters for their own preference ranks plus approval votes. Novel mathematical elements distinguish this paper. Its Condorcet completion methods examine all $\left(\begin{array}{c}m \\ 3\end{array}\right)$ candidate triples, sometimes analyze loop(s) of some of those triples, and order candidates in a set by first determining the last-place candidate. Its non-manipulability proofs involve mathematical induction on $m$.
\end{abstract}

Keywords: Condorcet completion methods; strategic voting; manipulable; cyclical majorities; approval voting; mathematical induction 


\section{Introduction}

This paper can be considered a complement to an earlier one (Potthoff [1], hereafter Po0). Both papers address the problem of devising single-stage voting systems to select a single winner from $m(>2)$ candidates, under the specific premise that a system should have two principal goals:

(i) to elect a Condorcet winner (a candidate who could win pairwise against each opponent) if one exists according to the profile of true preferences of the electorate, and

(ii) to be resistant to manipulation, with no lure to vote insincerely.

Both papers develop voting systems that thwart manipulations by sophisticated voters who strategically misrepresent their preferences so as to defeat a Condorcet winner. Thus, they both involve game-theory concepts. Our aim is to find voting systems where truthful revelation of preferences is an equilibrium strategy, leading to the election of the Condorcet winner, but in settings necessarily limited to electorate domains that exclude some cyclicities in true preferences.

To achieve (i) and (ii) together, both papers use unorthodox ballots (more so in the case of the present paper), and both of them assume and exploit voters' knowledge of electorate preferences (again more so in the present paper). Both papers utilize both candidate rankings and approval voting in certain ways.

For our purposes a voting system (or election system, or just system) is characterized by:

(1) its ballot;

(2) its procedure for aggregating the ballots to determine the winner; and

(3) a set $\mathrm{S}$ of sincere voting strategies that specifies how all voters are to mark their ballots, given their true preferences (i.e., as a function of those preferences).

The present paper covers voting systems with two types of ballots and three associated aggregation procedures for each. One ballot asks voters to rank the candidates with respect both to their own preferences and to their discerned preferences of the whole electorate. The second ballot is simpler and asks for voters' own preferences plus approval votes. Po0 likewise covered six systems based on two ballot types each with three aggregation procedures.

Compared with the systems of the present paper, those of Po0 are simpler (both practically and mathematically) and are more fit for large public elections, but have non-manipulability properties that are less strong. Thus, the voting systems of the two papers illustrate a tradeoff between non-manipulability and simplicity.

Beyond its use of ballots that are out of the mainstream and its exploitation of voters' knowledge of electorate preferences, the present paper is also distinctive because of several interesting mathematical features. Briefly, they include the following. The non-manipulability proofs use mathematical induction on $m$; such proofs do not usually do so. The ballot aggregation assigns a key role to the set of $\left(\begin{array}{c}m \\ 3\end{array}\right)$ candidate triples. In addition, special loops of some of those triples undergo auxiliary analyses in some cases. For the Condorcet completion method that has to be available (under any Condorcet system) to determine the winning candidate when the ballots show no Condorcet winner, a unique procedure applies. When $m>3$, that procedure successively, for $j=4, \ldots, m$, orders the candidates in each of the $\left(\begin{array}{c}m \\ j\end{array}\right) j$-tuples by first determining the last-place ( $j$-th place) candidate and then placing the remaining candidates in the same order as in the $(j-1)$-tuple that contains them all. Examples 1 and 2 in Section 4 
demonstrate basic details, for $m=5$ and 4 (respectively), and, like other, later examples, will aid in understanding the systems.

We use the letter $G$ to refer to a group composed of all voters sharing the same true-preference ranking of the $m$ candidates. We call $\mathrm{G}$ a preference group. (If there are $m=3$ candidates $\mathrm{X}, \mathrm{Y}$, and $\mathrm{Z}$, then (e.g.) $G(Z X Y)$ denotes the group of all voters who prefer $Z$ to $X$ to $Y$.) For a given domain of admissible true-preference profiles over the candidates, a voting system will be called non-manipulable if no group $\mathrm{G}$ can change the winner of the election to a candidate it prefers (not necessarily its top-preference candidate) by part or all of $\mathrm{G}$ deviating from the strategy set $\mathrm{S}$ for the system, given that no one else deviates from $\mathrm{S}$. Otherwise, the system is manipulable.

The notion here is basically a game-theory concept, where each group $G$ that shares a preference ranking corresponds to a player in a game, and where non-manipulability is equivalent to a Nash equilibrium. In most game-theory problems, however, the rules of the game are given, and the goal is to find the best strategy for each player. But in the choice of a voting system the scene is reversed: Our aim is to select the system (i.e., the rules of the game and the set $\mathrm{S}$ ) so that the strategy of each "player" (each G) is in accord with (i) and (ii) above, that is, leads to the election of the Condorcet winner and offers no advantage to unilateral deviation from $\mathrm{S}$ (where $\mathrm{S}$ is consistent with sincere voting). Note that "unilateral deviation" refers here to deviation by a group of voters all having the same ranking of true preferences, rather than deviation just by an individual voter.

Our assumed settings, covered in Section 5, are ones where cyclicities in true preferences are fully or partially ruled out: Our non-manipulability results are for domains of true-preference profiles that exclude those with any preference cycles at all (in some settings) or exclude only those that lack a Condorcet winner (in other settings). No such constraints apply, though, for any reported preferences (which may differ from true preferences if there is strategic voting).

The non-manipulability of the systems of this paper comes about because of the ballot property that asks voters to provide extra markings beyond the usual rankings on a ranked ballot. These added markings relate in one way or another to what we are calling discerned electorate preferences, or just electorate preferences, which refer to the profile of true preferences of the electorate as a whole. We postulate that the needed information about these electorate preferences is available to all voters (on a pragmatic level, see Remark 10.10 below in regard to possible sources of this information).

The additional ballot markings enable exploitation of the discerned electorate preferences and thereby enable use of Condorcet completion methods that render the strategic contrivance of vote cycles unrewarding. With only the usual voter rankings and without the added markings, manipulability is unavoidable, as Remark 10.18 and its example point out.

The function of the extra markings is to deter manipulation (see Remark 10.11). One might wonder, though, whether they could actually offer added opportunity for manipulation. But that type of manipulation (as well as all others) is already taken into account in our non-manipulability proofs.

Other Condorcet-consistent systems, such as those of Nanson, Kemeny, and Dodgson, are simpler for the voter than those of the present paper, so why are they not superior? They are more manipulable. In fact, for specific preference profiles each of these three is as easy to manipulate as the highly manipulable Borda system, as is illustrated at the end of Section 4.1 below.

In what follows, Section 2 deals with minor assumptions and with terminology. Ballots, aggregation procedures, and strategy sets are defined in Section 3. Section 4 provides the two examples mentioned 
earlier. Section 5 deals with non-manipulability and manipulability but relegates two mathematically complex non-manipulability proofs to Appendixes A and B. A manipulability comparison of some systems in this paper and Po0 is examined through an example in Section 6. Section 7 provides an aggregation shortcut, with mathematical proof in Appendix C. Circumvention of minor restrictions is the topic of Section 8 .

All parts of the paper just mentioned are strictly mathematical, in the sense that, given our assumptions, the conclusions are direct mathematical consequences. The assumptions themselves, however, may separately raise some questions, in both practical and philosophical terms. Concerns about the assumptions have no effect on the correctness of our mathematical results, but nonetheless need to be recognized. An example in Section 9 deals with a quandary that arises from uncertain discernment of electorate preferences. Then, in regard to the assumptions as well as the framework of the paper, Section 10 consists of various remarks that provide analytical discussion, supplementary comments, and numerous references. Section 11 concludes.

\section{Secondary Assumptions, and Terminology}

Some simplifying assumptions are needed in order to avoid excessive complexity, though Section 8 will show how they can be skirted. In any preference profile, it is assumed that no voter is indifferent between any two of the $m$ candidates (and that individual preferences are transitive). There are thus $m$ ! preference groups G. (We must deal with all $m$ ! of them, not just with the $m$ (coarser) groups whose members share the same top preference but otherwise differ.)

Regarding the ballots, we assume that each voter ranks all $m$ candidates using ranks from 1 to $m$ (with no ties) for the voter's own (reported) preferences on both types of ballot, as well as for the discerned electorate preferences on the first type of ballot. It is assumed that each voter provides the approval votes that are asked for on the second type of ballot.

In any preference tallies or vote tallies, it is assumed that no ties between candidates occur and that no total involving preferences or votes is exactly half the total number of voters. As needed, the random tie-breaking device described in Section 8 will ensure that this assumption holds if it does not already hold initially.

For clarity, we will henceforth refrain from using the term Condorcet winner, and will avoid as well the two-word terms Condorcet runner-up, Condorcet loser, Condorcet cycle, and cyclical majorities. In their place will be three-word terms obtained by inserting either preference or vote in the middle of a two-word term. A Condorcet preference winner is thus a candidate whom the electorate prefers to each opponent in a two-way match-up with each, and Condorcet vote winner is defined likewise but based on actual votes from a ranked ballot (i.e., reported preferences rather than true preferences). The no-ties assumptions simplify the meaning of a Condorcet preference winner or vote winner. Condorcet preference runner-up will refer to a candidate whom the electorate prefers to each other candidate except a Condorcet preference winner, and Condorcet vote runner-up is defined analogously. A candidate $\mathrm{X}$ is a Condorcet preference loser if the electorate prefers every opponent to X. Meanings of other three-word terms should be obvious. Sometimes a Condorcet preference cycle (where, e.g., majorities of the electorate prefer $\mathrm{X}$ to $\mathrm{Y}, \mathrm{Y}$ to $\mathrm{Z}$, and $\mathrm{Z}$ to $\mathrm{X}$ ) will simply be called a preference cycle, and similarly for a Condorcet vote cycle. 
The term basic ranking applies to both ballot types and will refer to the voter's reported preference ranking, whether sincere or not. It is the same as the voter's true preference ranking if the voter is non-strategic and votes sincerely.

For any preference group $\mathrm{G}$, the set $\mathrm{S}$ for a given voting system specifies the (sincere) voting strategy for $\mathrm{G}$. It will be called the base strategy for $\mathrm{G}$ under that system.

Our systems all make use of approval voting (Brams and Fishburn [2]) in one fashion or another. We use the term approval voting in a way that differs a bit in a nonmathematical sense, though not in a mathematical one, from its usual usage. Specifically, the term will simply connote (for any voter's ballot) a binary separation of candidates in a given set into those who receive a vote in that set and those who do not, without necessarily signifying either approval of the former or disapproval of the latter. We could have used a different term such as binary voting, but we keep the more familiar term.

\section{The Voting Systems}

The six systems considered in this paper are denoted by RR1, RR2, RR3, RA1, RA2, and RA3. The first three use the first ballot type; the other three, the second ballot type. The first three have three somewhat differing aggregation procedures, whereas the last three have a common aggregation procedure. On the other hand, there is a common set $\mathrm{S}$ of base strategies for the first three but three different sets for the last three. Sections 3.1-3.3 cover, respectively, the ballots, the aggregation procedures, and the sets of base strategies. The six systems can efficiently be described and dealt with together, but with attention to the special characteristics of each one.

\subsection{The Ballots}

The two types of ballots are called RR and RA ballots and are used, respectively, in the RR1, RR2, RR3 and RA1, RA2, RA3 election systems. The RR ballot asks the voter to provide two different rankings of the $m$ candidates. The first is the basic ranking (reported preference ranking). The second is the discerned electorate-preference ranking, or simply electorate ranking, for which a voter is asked to rank the candidates based on knowledge of the preference ordering of the entire electorate. Section 3.3 explains this second ranking further.

Instead of two rankings, the RA ballot asks for just one: the basic ranking. But it also asks for approval votes. They are subject to the restriction (corresponding to what Brams and Fishburn [2] call sincere approval voting) that one cannot cast an approval vote for any candidate $\mathrm{X}$ unless one also casts an approval vote for every candidate who appears higher than X on one's basic ranking. Ranked voting and approval voting are thus combined on the same ballot. The RA ballot (unlike that for RR) is not really new; it is much the same as the one that Nurmi [3] (p. 176) described. (It also is the same as the ballot for the RA* systems covered in Po0.)

\subsection{The Aggregation Procedures}

Under any of the six election systems, if there is a Condorcet vote winner (as determined from the basic rankings on the ballots), then the system simply chooses that person as the winning candidate and concludes. If not, the aggregation procedure invokes a Condorcet completion method. Because these 
methods (covered next) are complicated, the reader may find it helpful to look at the two examples in Section 4 in conjunction with studying the rest of Section 3.

\subsubsection{To Start: Ascribe Approval Votes within Each Candidate Triple}

There are $\left(\begin{array}{c}m \\ 3\end{array}\right)$ possible candidate triples that can be chosen from the $m$ candidates. For each voter's ballot for each triple, each election system ascribes an approval vote to either one or two of the three candidates in the triple. The aggregation procedures for the six systems differ only with respect to the rules by which the Condorcet completion methods ascribe these approval votes, which are captured at the outset. Along with the candidate triples themselves, the ascribed approval votes play a complex but key role that enables the aggregation procedures to bring about non-manipulability properties for the systems, as will be established through later proofs.

For a given individual ballot for a given triple, let B1, B2, and B3 denote (respectively) the candidates who (within the triple) are ranked highest (most favorable), second, and lowest on the basic ranking, and (for RR) let E1, E2, and E3 denote the candidates who are first, second, and third on the electorate ranking. The rules are as follows for the four aggregation procedures.

In the procedure for the RR1 system: For each ballot for each of the $\left(\begin{array}{c}m \\ 3\end{array}\right)$ triples, find whether B1 and $\mathrm{E} 1$ are the same candidate. If so, then (for this ballot for this triple) ascribe an approval vote to B1 only. If not, both $\mathrm{B} 1$ and $\mathrm{B} 2$ receive an approval vote.

RR2: If B1 and E3 are the same candidate, ascribe an approval vote to both B1 and B2. If not, only B1 gets an approval vote.

RR3: If $\mathrm{B} 1$ and $\mathrm{E} 1$ are not the same candidate and also B3 and E3 are not the same, then approval votes go to both $\mathrm{B} 1$ and $\mathrm{B} 2$. Otherwise, only $\mathrm{B} 1$ receives an approval vote.

RA1, RA2, and RA3: They all use the same rule for ascribing approval votes. For a given ballot for a given triple, ascribe an approval vote only to $\mathrm{B} 1$ if the ballot shows approval votes for none or one of the candidates, or to both B1 and B2 if it has approval votes for two or all three.

Illustration for RR. With four candidates, suppose that a ballot shows BDAC for the basic ranking and $\mathrm{ABCD}$ for the electorate ranking. Then, for that ballot, approval votes are ascribed for the $\mathrm{ABC}$ triple to both B and A under RR1, but only to B under RR2 and RR3; for the ABD triple to both B and D under RR1 and RR3, but only to B under RR2; for the ACD triple to both D and A under all three rules; and for the BCD triple to $\mathrm{B}$ alone under all three rules.

Illustration for RA. Suppose that a ballot shows a basic ranking of WXYZ for four candidates, with an approval vote for $\mathrm{W}$ only. Then an approval vote is ascribed to $\mathrm{X}$ (alone) in the XYZ triple. Ascribed approval votes go only to $\mathrm{W}$ in the other three triples.

\subsubsection{Steps in the Remainder of the Aggregation Procedure}

In the rest of the aggregation procedure, the Condorcet completion method is the same for all six systems. The steps are as follows. 
Step 1: A triple will be called cyclical if the basic rankings (upon tabulation across all ballots) reveal that its three candidates exhibit a Condorcet vote cycle. Otherwise, it is noncyclical. Find which of the $\left(\begin{array}{l}m \\ 3\end{array}\right)$ triples are cyclical and which are noncyclical.

Step 2: For each of the $\left(\begin{array}{l}m \\ 3\end{array}\right)$ triples, tally the total number of ascribed approval votes (see Section 3.2.1) across all ballots for each candidate in the triple.

Step 3: In each of the $\left(\begin{array}{c}m \\ 3\end{array}\right)$ triples, rank the candidates 1, 2, 3. If a triple is cyclical, rank the candidates based on their numbers of approval votes within the triple. If a triple is noncyclical, ranks 1, 2, and 3 go (respectively) to the Condorcet vote winner, runner-up, and loser. (Note: Step 2 obtains approval-vote totals for both cyclical and noncyclical triples. The totals for the latter are not used at this stage but will sometimes be required later.)

Steps $\mathbf{4}\{\mathbf{4}\}$ (groups of $j=4$ candidates), $\mathbf{4}\{\mathbf{5}\}(j=5), \ldots, \mathbf{4}\{\boldsymbol{m}\}(j=m)$ : These step(s) (applicable only when $m>3$ ) consist of ranking the candidates first in all $\left(\begin{array}{c}m \\ 4\end{array}\right)$ 4-tuples (quadruples) of candidates, then in all $\left(\begin{array}{c}m \\ 5\end{array}\right)$ 5-tuples, and so forth. To rank any of the $\left(\begin{array}{c}m \\ j\end{array}\right) j$-tuples $(j=4,5, \ldots, m)$, one first determines which candidate is to rank $j$-th (last), using the method given in Section 3.2.3 just below. Then the ranks of the remaining $(j-1)$ candidates are made the same as in the $(j-1)$-tuple that contains them all.

Step 5: The candidate ranked first in the $m$-tuple of all candidates (Step $4\{m\}$ if $m>3$ ) is declared the winner of the election.

\subsubsection{Determining the Lowest-Ranked Candidate}

Let $\mathrm{L}$ denote the candidate to be ranked last in a $j$-tuple. The method to find $\mathrm{L}$ may require as many as three (multi-step) operations, which are as follows:

(i) Consider the $j(j-1)$-tuples that are subsets of the $j$-tuple. $\mathrm{L}$ is taken as the candidate with the most $(j-1)$-th places in the $j \quad(j-1)$-tuples. If there is a tie, $\mathrm{L}$ is the one (among the tied candidates) with the most $(j-2)$-th places in the $j \quad(j-1)$-tuples. If there is still a tie, continue to try to break it by examining numbers of $(j-3)$-th, $(j-4)$-th, ..., 2 nd places among those candidates who at each point are still in the running to be chosen as L. If now a tie still persists, break it by using Method M1 if the tie is not among all $j$ candidates. (For more about M1 as well as two other methods to be mentioned shortly, M2 and M3, see Section 3.2.4 below.) If the tie is among all $j$ candidates, thus implying that in the $j \quad(j-1)$-tuples each candidate appears exactly once in each of the $(j-1)$ places, then go on to (ii).

In Example 1 in Section 4, the aggregation procedure is able to terminate after applying operation (i) first in Step $4\{4\}$ (five times, with $j=4$ ) and then in Step $4\{5\}$ (once, with $j=5=m$ ).

(ii) If any candidate $\mathrm{X}$ has the same candidate ( $\mathrm{Y}$, say) ranked immediately below $\mathrm{X}$ in all $(j-2)$ of the $(j-1)$-tuples in which both appear, then call $\mathrm{X}$ a capping candidate. It will be said that $\mathrm{X}$ caps Y. Determine if all candidates are capping candidates. If not, use Method M2 to choose L from those candidates who are not capping candidates. If so, go on to (iii).

(iii) Because all $j$ candidates are capping candidates, a single loop comprising all of them is automatically defined. In this loop, candidate $i_{1}$ caps $i_{2}$, $i_{2}$ caps $i_{3}, \ldots, i_{j-1}$ caps $i_{j}$, and $i_{j}$ caps $i_{1}$, where the candidates are numbered with $j$ of the integers from 1 through $m$ and $\left(i_{1}, i_{2}, \ldots, i_{j}\right)$ is some permutation of those $j$ integers. (For $j=6$, e.g., the set of six ordered quintuples in (i) and (ii) would have to be of the form ABCDE, BCDEF, CDEFA, DEFAB, EFABC, FABCD to reach operation (iii), so the loop would be $\mathrm{A}, \mathrm{B}, \mathrm{C}, \mathrm{D}, \mathrm{E}, \mathrm{F}$, and back to A.) Now consider the $j$ triples that consist of three consecutive 
candidates in the loop, and call them contiguous triples. If $(\mathrm{X}, \mathrm{Y}, \mathrm{Z})$ is a contiguous triple, with $\mathrm{X}$ capping $\mathrm{Y}$ and $\mathrm{Y}$ capping $\mathrm{Z}$, call $\mathrm{Y}$ the central member of the triple. For each contiguous triple, find the number of approval votes won by its central member. (Capture this number regardless of whether the triple is noncyclical or cyclical.) A candidate $\mathrm{Y}$ will be called a lesser candidate if, in the contiguous triple of which $\mathrm{Y}$ is the central member, Y's number of approval votes is less than half the total number of voters. Use Method M3 to choose L from among all lesser candidates, or from among all $j$ candidates if there is no lesser candidate.

Example 2 in Section 4 can stop only after operation (iii) (in Step $4\{4\}$, with $j=4=m$ ).

\subsubsection{Methods M1, M2, and M3}

Methods M1, M2, and M3 are not relevant to the non-manipulability proofs given later, so from that standpoint it is immaterial how they are specified. Thus, they could suitably be defined in numerous ways since they do not affect the proofs. For simplicity, all three can be made the same. One reasonable way for M1, M2, or M3 to choose $\mathrm{L}$ from $k$ of the $j$ candidates (where $k$ is the number who qualify to be chosen) is as follows. If $2<k<j$, let $\mathrm{L}$ be the candidate who ranks last in the $k$-tuple that consists of those $k$ candidates. If $k=2$, let $\mathrm{L}$ be whichever candidate is rated lower by more than half the voters in the basic rankings. If $\mathrm{L}$ has to be chosen from all $j$ candidates under consideration (not applicable for M1), let $\mathrm{L}$ be the candidate with the smallest number of approval votes totaled across the $\left(\begin{array}{l}j \\ 3\end{array}\right)$ subset triples.

\subsubsection{A Full Ordering Sometimes}

Although Section 3.2 aims to produce only a winner of the election, in some cases it also yields, as a by-product, a full ordering of the $m$ candidates. That occurs if either there are no Condorcet vote cycles or there is no Condorcet vote winner. It does not occur in the intermediate case where there is a Condorcet vote winner but Condorcet vote cycle(s) exist otherwise.

\subsection{The Sets of Base Strategies}

As noted earlier, the three RR systems have a common set $\mathrm{S}$ of base strategies, but the RA systems have three different sets $\mathrm{S}$. Under each of the six systems, the set S specifies simply that voters are to mark their basic rankings sincerely, identically to their true preferences. For marking the electorate ranking for the RR systems and the approval votes for the RA systems, though, the base strategies are more involved.

For RR1, RR2, and RR3, S directs voters to mark their electorate rankings in accord with their discernment of the preference profile of the entire electorate. Thus, if there are no Condorcet preference cycles, then every voter's electorate ranking assigns top rank to the (discerned) Condorcet preference winner, second rank to the Condorcet preference runner-up, and so on, with bottom rank going to the Condorcet preference loser.

If there is a Condorcet preference winner (call it A) but preference cycle(s) otherwise, then the voter is to rank $\mathrm{A}$ at the top of the electorate ranking. For the rest of this ranking, though, $\mathrm{S}$ can allow full leeway: Having A at the top suffices for the non-manipulability proof for RR1; RR2 and RR3 have no such proof for this case anyway. (The start of Section 5 below indicates the preference domain for each non-manipulability result.) Nonetheless, for definiteness one could define $\mathrm{S}$ further, such as by 
specifying that voters are to rank candidates within any preference cycle in accord with their own true preferences (basic rankings) but rank candidates outside such cycle(s) in accord with the discerned electorate preferences. Example 3 below follows that route.

For both RR and RA, we ignore for present purposes the case where there is no Condorcet preference winner. No non-manipulability results exist for that case.

For RA1, RA2, or RA3, the sets S direct voters to mark their approval votes using, respectively, Rule 1, 2, or 3 below. Let A denote the Condorcet preference winner and B the runner-up. (B is needed for Rules 2 and 3 only. If $B$ fails to exist, no non-manipulability proof is affected, since preferences are then outside the domain of non-manipulability for RA2 or RA3; but for definiteness S could specify, e.g., that Rules 2 and 3 default to Rule 1 if B does not exist.) The three rules (also cf. Section 3.3 of Po0) are as follows for voters in a given preference group G:

Rule 1 (RA1): Approval votes go to all candidates except $\mathrm{A}$ if $\mathrm{A}$ is the least preferred candidate of the $G$ voters, or to A and every candidate (if any) whom $G$ prefers to A otherwise.

Rule 2 (RA2): Approval votes go to A and to every candidate whom $G$ prefers to A if $G$ prefers $A$ to $B$, or only to $B$ and every candidate whom $G$ prefers to $B$ if $G$ prefers $B$ to $A$.

Rule 3 (RA3): Approval votes go to $A$ and to every candidate whom $G$ prefers to $A$ if $G$ prefers $A$ to $B$, or to every candidate whom $G$ prefers to $A$ if $G$ prefers $B$ to $A$.

It is easy to verify that, if there are only $m=3$ candidates, then the three rules just given for the RA systems are equivalent, respectively, to the three rules for ascribed approval votes stated in Section 3.2.1 for RR1, RR2, and RR3. Note, though, that the rules apply to the aggregation procedures in Section 3.2.1, whereas here they apply to the base strategies.

\section{Two Illustrations of How Manipulation Attempts Would Fail—Even Backfire}

\subsection{An Illustration with Five Candidates}

The following example, with 100 voters, shows how the six systems foil manipulation when there are $m=5$ candidates (labeled A through E):

\section{Example 1.}

\begin{tabular}{lccc}
\hline Group & G(ABCDE) & G(EDCAB) & G(BACDE) \\
\hline Number of voters & 35 & 25 & 40 \\
\hline RR1, RR2, RR3 vote & ABCDE/ABCDE & EDCAB/ABCDE & BCDEA/ABCDE \\
\hline RA1, RA2, RA3 vote & A|BCDE & EDCA|B & BCDE|A \\
\hline
\end{tabular}

In the RR vote row, the basic ranking is before the slash (/) and the electorate ranking is after. In the basic rankings in the RA vote row, the candidates before the vertical line $(\mid)$ are the ones who receive approval votes. Obviously, even though the example shows all six systems, no two would be applied simultaneously. The preference profile has no Condorcet preference cycles. The preference order of the electorate is $\mathrm{ABCDE}$, so $\mathrm{A}$ is the Condorcet preference winner.

$\mathrm{G}(\mathrm{ABCDE})$ and $\mathrm{G}(\mathrm{EDCAB})$ each follow their base strategies (for both the basic and electorate rankings) for the RR systems. For the RA systems each one also follows its base strategy, which (for 
each group) happens to be the same regardless of whether Rule 1 (for RA1), 2 (RA2), or 3 (RA3) is used.

But for both RR and RA, G(BACDE) voters deviate from their base strategy and mark their basic rankings in the classic way that can depose $\mathrm{A}$ under the Borda method (i.e., by ranking $\mathrm{A}$, the Condorcet preference winner, at the bottom, even though $\mathrm{A}$ is their second preference). For RR these voters do follow their base strategy for the electorate ranking (though they could not have dethroned A even if they had done otherwise, per Proposition 2 in Appendix A).

To start, consider RR. After first finding that there is no Condorcet vote winner, the aggregation procedure for each of the three RR systems (see Section 3.2) examines the $\left(\begin{array}{l}5 \\ 3\end{array}\right)=10$ triples. The ordering for the noncyclical triples, and the ordering and the approval-vote tallies for the cyclical triples (with one set of results for RR1 and RR3, another for RR2), are as follows:

\begin{tabular}{ll}
\hline Noncyclical triples: & CDA, CEA, DEA, BCD, BCE, BDE, CDE \\
\hline Cyclical triples, RR1 and RR3: & C 65, A 60, B 40; D 65, A 60, B 40; E 65, A 60, B 40 \\
\hline Cyclical triples, RR2: & A 60, B 40, C 25; A 60, B 40, D 25; A 60, B 40, E 25 \\
\hline
\end{tabular}

The first cyclical triple (e.g.) draws approval votes from $\mathrm{G}(\mathrm{ABCDE})$ for $\mathrm{A}$; from $\mathrm{G}(\mathrm{EDCAB})$ for $\mathrm{C}$ and $\mathrm{A}$; and from $\mathrm{G}(\mathrm{BACDE})$ for $\mathrm{B}$ and $\mathrm{C}$ under RR1 or RR3, but only for B under RR2.

The next step is to examine the $\left(\begin{array}{l}5 \\ 4\end{array}\right)=5$ quadruples of candidates. One finds the last-place candidate (L) in each quadruple by looking at its four subset triples. Then one puts the remaining three candidates (all except L) at the top of the quadruple, in the same order as in the triple that contains them all. The ordering of each quadruple and of its subset triples, along with its L (in each case here, the candidate who places third in the greatest number of triples), is as follows:

\begin{tabular}{ll}
\hline & CDAB (CAB, DAB, CDA, BCD; B is L) \\
For RR1 and RR3: & CEAB (CAB, EAB, CEA, BCE; B is L) \\
& DEAB (DAB, EAB, DEA, BDE; B is L) \\
\hline \multirow{3}{*}{ For RR2: } & ABCD (ABC, ABD, CDA, BCD; D is L) \\
& ABCE (ABC, ABE, CEA, BCE; E is L) \\
& ABDE (ABD, ABE, DEA, BDE; E is L) \\
\hline \multirow{2}{*}{ For all three: } & CDEA (CDA, CEA, DEA, CDE; A is L) \\
& BCDE (BCD, BCE, BDE, CDE; E is L) \\
\hline
\end{tabular}

The final step is to obtain the ordering of the set of all five candidates by examining the five subset quadruples. For RR1 and RR3, B has the most last places among these quadruples, so the ordering is $\mathrm{CDEAB}$. $\mathrm{E}$ has the most last places under RR2, so $\mathrm{ABCDE}$ is the ordering. Thus, the winner under RR2 is $A$, and under RR1 or RR3 is $C$ (even worse than $A$ for $G(B A C D E)$ ). In neither case does G(BACDE) dethrone $\mathrm{A}$ in favor of $\mathrm{B}$, the only candidate whom it prefers to $\mathrm{A}$.

Now consider RA. The example has been so constructed that the entire sequence of results under the RA aggregation procedure is identical with that just given for RR1 and RR3. In addition, if the deviating $\mathrm{G}(\mathrm{BACDE})$ voters were to vote $\mathrm{B} \mid \mathrm{CDEA}$ rather than $\mathrm{BCDE} \mid \mathrm{A}$ with all else unchanged, then the sequence of results would be the same as that for RR2 above.

Manipulation is curbed under our systems but, as it turns out in this example, is simple under not only the Borda method but also (e.g.) the Coombs method and the Condorcet methods of Nanson, Kemeny, 
and Dodgson. Easy manipulability of these last five is evident if one looks at Example 1 with D and E removed (for definitions of these five methods, see, e.g., Brams and Fishburn [4] and Tideman [5]).

Thus, $35 \mathrm{ABC}$ votes come from $\mathrm{G}(\mathrm{ABC})$ and $25 \mathrm{CAB}$ votes from $\mathrm{G}(\mathrm{CAB})$. Under any of the five methods, the $40 \mathrm{G}(\mathrm{BAC})$ voters then find it simple to manipulate by voting $\mathrm{BCA}$, thereby dethroning the Condorcet preference winner, A, in favor of their top preference, B. (With all the candidates retained as in Example 1 itself, the manipulated BCDEA votes by G(BACDE) still succeed in electing $\mathrm{B}$ under each of the five methods, but verifying that is less easy.)

\subsection{An Illustration of How the Loop of Candidate Triples Works}

The following example uses RR2 for illustration and shows how, in the aggregation procedure, the formation of a loop of candidate triples and the resort to identifying lesser candidates (see Section 3.2.3) protect the Condorcet preference winner from dethronement:

\section{Example 2.}

\begin{tabular}{lccc}
\hline Group & G(ABCD) & G(DCAB) & G(BACD) \\
\hline Number of voters & 35 & 25 & 40 \\
\hline RR vote & ABCD/ABCD & DCAB/ABCD & BCDA/ADBC \\
\hline
\end{tabular}

There are no preference cycles, and the preference order of the electorate is $A B C D . G(A B C D)$ and G(DCAB) each follow their RR base strategy, but G(BACD) does not.

After ascertainment that no Condorcet vote winner exists, examination of the $\left(\begin{array}{l}4 \\ 3\end{array}\right)=4$ triples yields the following results under the RR2 aggregation procedure:

\begin{tabular}{ll}
\hline Noncyclical triples: & CDA, BCD \\
\hline Cyclical triples: & A 60, B 40, C 25; D 65, A 60, B 40 \\
\hline
\end{tabular}

Because each of the four candidates has one third place, one second place, and one first place in the four triples, the process to determine L (the candidate who is to rank last in the quadruple) has to go on to another operation, which is to find any candidates who are not capping candidates.

But all four candidates are capping candidates (A caps B, B caps C, C caps D, and D caps A). There is thus a loop of the candidates (A, B, C, D, then back to A), which, in turn, generates a loop of contiguous candidate triples (DAB, ABC, BCD, CDA).

In the final operation, one finds which candidates are lesser candidates. In the two noncyclical triples, the total approval votes for their central members are 65 in CDA for D, and 25 in BCD for C. Thus, the approval-vote tallies for all four candidates from the triples of which they are the central members are 60 for $\mathrm{A}$ (from $\mathrm{DAB}$ ), 40 for $\mathrm{B}$ (from $\mathrm{ABC}$ ), 25 for $\mathrm{C}$ (from $\mathrm{BCD}$ ), and 65 for $\mathrm{D}$ (from CDA). The conclusion is that $\mathrm{B}$ and $\mathrm{C}$ are lesser candidates (because their approval votes are less than half the total of 100) whereas A and D are not.

Thus, depending on how Method M3 is defined, it chooses L to be either B or C. The ordering of the quadruple is $\mathrm{CDAB}$ in the former case or $\mathrm{DABC}$ in the latter, giving rise to $\mathrm{C}$ or $\mathrm{D}$ as the winning candidate. With either outcome, the manipulation attempt by G(BACD) backfires.

With the other five systems just as with RR2, one can construct examples, with no preference cycles and $m=4$, where one has to execute the entire aggregation procedure and examine a loop of four 
candidate triples (after which the unilateral manipulation is shown to fail). Thus, the routine that resolves the loop of triples, though complex, is key to deterring manipulation.

\section{Results on Non-Manipulability and Manipulability}

We examine whether the six systems of this paper are manipulable for each of three different domains of true-preference profiles of the electorate. In each domain, every profile has a Condorcet preference winner. Let F0 denote the domain of profiles where there are no preference cycles among the $m$ candidates. Let F1 denote the domain where there is a Condorcet preference winner but where preference cycles(s) may (or may not) exist otherwise. Note that F0 is a subset of F1. F0 and F1 are the same for $m=3$, of course. The three domains are F0 (or F1) with $m=3$, F0 (with $m \geq 3$ ), and F1 (with $m \geq 3$ ).

The largest domains of true-preference profiles for which non-manipulability will be proved are F1 for RR1 and RA1; F0 for RR2 and RR3; and F0 (or F1) just with $m=3$ for RA2 and RA3. The non-manipulability proofs are in Section 5.1 and the appendixes. Counterexamples in Section 5.2 show the manipulability of RR2 and RR3 in F1 and of RA2 and RA3 in F0.

\subsection{Non-Manipulability Proofs}

Right after an initial lemma (Lemma L1), Proposition 1 is stated and proved below. It establishes that all six systems are non-manipulable when there are only $m=3$ candidates (with a Condorcet preference winner). Propositions 2 and 3 have lengthy proofs and are in Appendixes A and B, respectively. The former states that RR1, RR2, and RR3 are non-manipulable in F0; the latter, that RR1 and RA1 are non-manipulable in F1. Lemma L1 and Proposition 1 constitute essential stepping-stones for the proofs in the appendixes.

Proposition 2 as it pertains to RR1 (but not to RR2 or RR3) may be seen as superfluous in view of Proposition 3. Proposition 2 seems to have a bit more straightforward proof than Proposition 3, though, and can include RR1 in its proof with virtually no added details.

When the statement that candidate $\mathrm{X}$ places above candidate $\mathrm{Y}$ is made in the rest of the paper, it will apply within a specific set of candidates. It will mean that $\mathrm{X}$ is ranked above $\mathrm{Y}$ by a step in the aggregation procedure that ranks the candidates for that particular set. Similarly, the statement that $\mathrm{X}$ places last in a set will mean simply that the aggregation procedure ranks $\mathrm{X}$ last in that set. The statement that $\mathrm{X}$ places above $\mathrm{Y}$ does not necessarily mean that the basic rankings show $\mathrm{X}$ rated above $\mathrm{Y}$ by more than half the voters, or necessarily reflect anything about any approval votes that may be tallied. It is possible for $\mathrm{X}$ to place above $\mathrm{Y}$ in one set of candidates that includes them both but for $\mathrm{Y}$ to place above $\mathrm{X}$ in another.

Lemma L1. Let there be $m=3$ candidates A, B, and C who are, respectively, the Condorcet preference winner, runner-up, and loser. Under any of the systems RR1, RR2, or RR3, let S1 denote the set of base strategies, which is the set of ballot strategies under which all voters mark their basic rankings according to their own true preferences, and mark their electorate rankings as A first, B second, and C third. Under the systems RA1, RA2, and RA3, let S2 denote the set of ballot strategies (again base strategies) under which all voters mark their basic rankings according to their own true preferences, and mark their approval votes in accord with Rules 1, 2, and 3, respectively. For any of the six systems, suppose that all 
preference groups other than $\mathrm{G}(\mathrm{BAC})$ follow $\mathrm{S} 1$ or $\mathrm{S} 2$ (as applicable) but that $\mathrm{G}(\mathrm{BAC})$ can deviate from S1 or S2. Then both of the following hold no matter what ballot strategy G(BAC) adopts:

L1.1: If approval votes are tallied in accordance with any of the six systems (whether or not the three candidates show cyclical vote majorities that trigger such a tally), then A will receive approval votes from more than half the electorate and $\mathrm{B}$ from less than half.

L1.2: Within the set of three candidates, A will place above B.

Remark. With just three candidates there would be no occasion to tally approval votes unless cyclical vote majorities arise in the tally of the basic rankings. L1.1 is worded broadly, however, for use in later proofs where approval votes do have to be tallied within a subset of three out of $m(>3)$ candidates even when cyclical vote majorities do not occur in that subset.

Proof of L1.1. Regardless of whether an RR or RA system is in effect and regardless of which of the three rules for ascribing or marking approval votes applies, candidate A will receive approval votes from $\mathrm{G}(\mathrm{ABC}), \mathrm{G}(\mathrm{ACB})$, and $\mathrm{G}(\mathrm{CAB})$, and $\mathrm{B}$ will receive no approval votes from them. Because these three preference groups are the ones that prefer $A$ to $B$, they make up more than half the electorate. G(BAC) can thus do nothing to forestall the conclusion stated in L1.1.

Proof of L1.2. If the ballot strategy of $\mathrm{G}(\mathrm{BAC})$ causes cyclical vote majorities to occur, then A will get more approval votes than B by virtue of L1.1 and will thus place above B. If not, then A will still place above $B$ because the basic rankings on more than half the ballots - those from $G(A B C), G(A C B)$, and $\mathrm{G}(\mathrm{CAB})$ - will show A above B.

Proposition 1. Let there be $m=3$ candidates with the same conditions and notation as in the first three sentences of Lemma L1. (Among other things, the domain for admissible true-preference profiles is thus F0, or F1, with $m=3$.) Then each of the systems RR1, RR2, RR3, RA1, RA2, and RA3 is non-manipulable. That is, no preference group $\mathrm{G}$, by deviating from S1 or S2 when no one else does, can adopt a ballot strategy that will dethrone A (the Condorcet preference winner) as the election winner in favor of a candidate whom $G$ prefers to $A$.

Proof (similar to that of Proposition 3 in Po0, p. 106). For each of the six preference groups, one needs to show that that group can gain nothing by deviating from S1 or S2 (whichever one is applicable), given that the other five groups all adhere to $\mathrm{S} 1$ or $\mathrm{S} 2$. The following statements apply to all six systems. $\mathrm{G}(\mathrm{ABC})$ or $\mathrm{G}(\mathrm{ACB})$ voters cannot gain by deviating, because their most preferred candidate (A) already wins. Because $\mathrm{G}(\mathrm{BCA})$ or $\mathrm{G}(\mathrm{CBA})$ voters are already marking A last in their basic rankings under $\mathrm{S} 1$ or $\mathrm{S} 2$, they can adopt no ballot strategy that would prevent $\mathrm{A}$ from being the Condorcet vote winner. Because $\mathrm{G}(\mathrm{CAB})$ voters are already marking $\mathrm{C}$ first in their basic rankings under S1 or S2, they can do nothing to stop $\mathrm{C}$ from being the Condorcet vote loser; thus, they can achieve nothing better than the election of A, their second preference. Finally, G(BAC) voters can likewise achieve nothing better than the election of their second preference (A), because, by L1.2, they cannot prevent B from placing below A regardless of whether they do or do not bring about cyclical vote majorities.

Remark. Brams and Sanver [6] showed that, under approval voting with voting assumed to be sincere, there exists with respect to a Condorcet preference winner a (rather stringent) specific set of strategies, the "critical strategy profile," under which no coalition of preference groups can dislodge that winner in favor of a candidate whom everyone in the coalition prefers. That is, this is a strong Nash equilibrium (for any $m$ ), not just a Nash equilibrium pertaining to manipulation by a single preference group. The present paper concentrates on systems that inhibit manipulation by an individual preference 
group (rather than by a coalition of voters with differing preference rankings, which would entail far greater complexity). One might ask, though, whether the various Nash equilibria that our systems enjoy are also strong Nash equilibria. For $m=3$ candidates, an argument a bit more general than the one in the proof of Proposition 1 shows that the answer is yes for RR1 and RA1. But for the other four systems, the answer is no even for $m=3$, as can be shown by suitable minor modification of Example 3 of Po0.

\subsection{Counterexamples that Show Manipulability}

The following example shows that RR2 and RR3 are manipulable in F1:

\section{Example 3.}

\begin{tabular}{llll}
\hline Group & G(CADB $)$ & G(DABC) & G(BACD) \\
\hline Number of voters & 35 & 25 & 40 \\
\hline RR2 or RR3 vote & CADB/ACDB & DABC/ADBC & BCDA/BCDA \\
\hline
\end{tabular}

Although $\mathrm{A}$ is the Condorcet preference winner, there is a preference cycle among $\mathrm{B}, \mathrm{C}$, and $\mathrm{D}$ (B preferred to C, C to D, D to B). Thus, the domain is F1. The vote of each group except G(BACD) is consistent with the base strategy for RR2 and RR3 (and RR1) as specified in Section 3.3: All voters in these two groups mark their basic rankings in accord with their true preferences and put $\mathrm{A}$ at the top of their electorate rankings.

There is no Condorcet vote winner, thanks to manipulation by G(BACD). The following results for the four triples apply for either RR2 or RR3:

\begin{tabular}{ll}
\hline Noncyclical triples: & CDA, DAB \\
\hline Cyclical triples: & B 40, C 35, A 25; B 40, C 35, D 25 \\
\hline
\end{tabular}

Because A has the most last places in the triples, A places last in the quadruple. The resulting ordering of the quadruple, BCDA, renders successful the efforts of $G(B A C D)$ to dethrone $A$ in favor of candidate B.

Note that the manipulation attempt fails under RR1 (as it must, per Proposition 3 in Appendix B). RR1 changes the ordering of the cyclical triple $\mathrm{BCA}$ to $\mathrm{ABC}$, because 35 added approval votes are ascribed to A from $\mathrm{G}(\mathrm{CADB})$. A loop of the four candidates now results. Under RR1 their approval votes from the triples in which they are the central members are then 60 for $\mathrm{A}$ (from $\mathrm{DAB}$ ), 40 for $\mathrm{B}$ (from $\mathrm{ABC}$ ), 35 for $\mathrm{C}$ (from $\mathrm{BCD}$ ), and 25 for $\mathrm{D}$ (from $\mathrm{CDA}$ ). B, C, and D (but not A) are thus lesser candidates. No matter which of the three Method M3 chooses to be last in the quadruple, the ploy by $\mathrm{G}(\mathrm{BACD})$ is unrewarded because B will not be first in the quadruple.

Example 3 bears an interesting resemblance to Example 2 of Peress [7]. Although the voting system ("multi-stage runoff") for his example is totally different from RR2 or RR3, the two preference profiles are essentially the same and the results in both cases reflect anomalies when there is a Condorcet preference winner but preference intransitivity otherwise.

The second counterexample shows that RA2 and RA3 can each be manipulated when there are $m=4$ candidates without any cyclical preference majorities (domain F0): 


\section{Example 4.}

\begin{tabular}{lccccc}
\hline Group & G(ABCD) & G(DCBA) & G(DABC) & G(DBAC) & G(CABD) \\
\hline Number of voters & 43 & 40 & 4 & 4 & 9 \\
\hline RA2 or RA3 vote & $\mathrm{A} \mid \mathrm{BCD}$ & $\mathrm{DCB} \mid \mathrm{A}$ & $\mathrm{DA} \mid \mathrm{BC}$ & $\mathrm{DB} \mid \mathrm{AC}$ & $\mathrm{C} \mid \mathrm{DAB}$ \\
\hline
\end{tabular}

$A B C D$ is the preference order of the electorate. The votes shown for each group except $G(C A B D)$ follow base strategy, under either RA2 or RA3. G(CABD) prevents a Condorcet vote winner from emerging.

The RA aggregation procedure produces the following results for the four triples:

\begin{tabular}{ll}
\hline Noncyclical triples: & ABC, DAB \\
\hline Cyclical triples: & C 49, D 48, A 47; C 49, D 48, B 47 \\
\hline
\end{tabular}

Because $\mathrm{B}$ has the most third places (two) in the triples, $\mathrm{B}$ is last in the quadruple, thereby causing the ordering of the quadruple to be CDAB. Thus, the manipulation by $\mathrm{G}(\mathrm{CABD})$ succeeds in dethroning $\mathrm{A}$ in favor of $\mathrm{C}$, its first preference.

Observe that, with RA1, the base strategy changes for one of the groups: G(DBAC) votes DBA|C rather than $\mathrm{DB} \mid \mathrm{AC}$. That change causes $\mathrm{A}$ to receive 51 approval votes instead of 47 in the first cyclical triple, thus averting the defeat of A by $\mathrm{C}$. Any kind of manipulation attempt by G(CABD) under any of the RR systems will likewise fail (per Proposition 2 in Appendix A).

\section{RA* (from Po0) versus RA}

The RA* ballot of Po0 is the same as the RA ballot of this paper. The systems $\mathrm{RA}^{*} 1, \mathrm{RA} * 2$, and RA*3 of Po0 use, respectively, the same base strategies as RA1, RA2, and RA3. The RA* aggregation procedure, though, is different: If there is no Condorcet vote winner, all RA* systems just choose the election winner via a straight tally of approval votes. But possible greater manipulability of those systems may counteract their greater simplicity. In the following example, which further develops Example 2 of Po0, RA*2 and RA*3 are manipulable whereas RA2 and RA3 are not:

\section{Example 5.}

\begin{tabular}{lllll}
\hline Group & G(ABCD) & G(BACD) & G(DABC) & G(CABD) \\
\hline Number of voters & 7 & 27 & 29 & 37 \\
\hline RA2, RA3, RA*2, or RA*3 vote & A|BCD & B|ACD & DA|BC & C|DBA \\
\hline
\end{tabular}

There are no preference cycles, and ABCD is the preference order of the electorate. For each preference group above, all four systems happen to prescribe the same base strategy. Each group except $\mathrm{G}(\mathrm{CABD})$ follows its base strategy. But thanks to the manipulation by $\mathrm{G}(\mathrm{CABD})$, no Condorcet vote winner emerges. With RA*, the ensuing count of approval votes yields 36 for A, 27 for B, 37 for C, and 29 for D. Thus, $\mathrm{C}$ wins and the manipulation succeeds.

But under RA, the manipulation fails. The ordering of the two noncyclical triples, and the ordering and approval votes for the two cyclical triples, are as follows:

\begin{tabular}{ll}
\hline Noncyclical triples: & BAC, DBA \\
\hline Cyclical triples: & A 63, C 37, D 29; C 37, B 34, D 29 \\
\hline
\end{tabular}


Note that, despite the locations of the vertical lines, A's approval votes include 27 from G(BACD) for the ACD triple, and $B$ gets seven votes from $G(A B C D)$ for the $C B D$ triple. $D$ is last in the quadruple because D has the most last places (two) in the four triples. Thus, the order of the quadruple is BACD, B wins, and B's victory is not favorable to G(CABD).

Conceivably one could discover an example where manipulation succeeds with RA2 (or RA3) but fails with RA*2 (or RA*3). Such a case has not been found, however.

For RA and RA* to produce a difference, $m$ must be $\geq 4$. The RA and RA* systems are effectively the same when $m=3$.

\section{Simplification of the Aggregation Procedures}

All the Condorcet completion methods in this paper (see Section 3.2) have been defined in terms of applying them to all $m$ candidates when there is no Condorcet vote winner. A shorter procedure is possible. An obvious - and simpler - approach would be to apply the Condorcet completion method (if it has to be invoked) to just the candidates in the top vote cycle. The top vote cycle is defined to be the smallest set of candidates such that, in the basic rankings, each candidate in the set is ranked above each candidate outside the set by a majority of voters. For all six of our election systems (with their four aggregation procedures), the winner of the election will be the same if the Condorcet completion method is applied just to the candidates in the top vote cycle rather than to all $m$ candidates. This is shown by Proposition 4, proved in Appendix C.

\section{Circumventing the Minor Assumptions}

The minor simplifying assumptions that are stated in Section 2 involve both preferences and votes, thus entailing theoretical and operational concerns, respectively. All of these assumptions, however, can be circumvented if they are not satisfied.

A ballot that is incompletely marked violates the assumptions. It can be resolved by treating it as $v$ separate ballots, each with weight $1 / v$, where $v$ is the number of possible ways of completing the ballot and one of the $v$ ballots corresponds to each of these $v$ ways. Thus (e.g.), if an RR ballot with candidates $\mathrm{X}, \mathrm{Y}$, and $\mathrm{Z}$ has $\mathrm{Z}$ ranked first on the basic ranking, $\mathrm{X}$ first on the electorate ranking, and no other rankings marked, it can be treated as $1 / 4$ of a ballot for each of ZXY/XYZ, ZXY/XZY, ZYX/XYZ, and ZYX/XZY. If (e.g.) an RA ballot with four candidates shows $Y \mid W$ but leaves $X$ and Z unranked below $Y$ and $W$, it can be treated as $1 / 2$ of a ballot for each of $Y \mid W X Z$ and $Y \mid W Z X$, but it can be treated as 1/6 of a ballot for each of $Y|W X Z, Y| W Z X, Y W|X Z, Y W| Z X, Y W X \mid Z$, and YWZ|X if it shows just YW with no approval-vote marking.

Indifference in the preference rankings (another assumption violation) can be handled in essentially the same way as an incompletely-marked ballot. Thus (e.g.), with four candidates a voter who prefers $\mathrm{W}$ most and $X$ least but is indifferent between $Y$ and $Z$ can be treated as $1 / 2$ of a voter for each of the WYZX and WZYX preference rankings.

Finally, the assumptions precluding ties and the like can be readily circumvented. Let $n$ be the total number of voters in the electorate. Let $u_{g}(g=1, \ldots, n)$ be independent random variables each drawn from the uniform (rectangular) distribution with range from 0 to 1 . Now suppose that each voter $g$ receives a weight of $\left(1+u_{g} / n\right)$ instead of 1 . Such slightly differing weights for the voters automatically produce a 
random tie-breaking mechanism. It ensures that vote tallies and preference tallies will both satisfy the assumptions of no ties between candidates and no totals exactly equal to $n / 2$. Moreover, as one can easily show, the differential weighting will give the same results as the equal weighting when there is no assumption failure under equal weighting.

Breaking ties randomly is, in general, a standard approach (cf. Pritchard and Wilson [8] (p. 489)). Ties are less likely the more voters there are, of course.

\section{Are RR1 and RA1 Still Best if the Information Postulate Is Relaxed?}

Thus far, this paper has been purely mathematical. We now turn to broader issues.

Treating voters more as real-world people and less as mathematical entities, we first illustrate the effect of uncertainty about electorate preferences. Because RR1 and RA1 are non-manipulable in F1 whereas the other four systems are non-manipulable only in F0 (and just for $m=3$ for RA2 and RA3), it would appear that RR1 and RA1 are the only systems that merit consideration. That is the case if the postulate stipulating the needed knowledge of electorate preferences strictly holds. But if not, the other four systems are, in some cases, better at avoiding pitfalls stemming from incorrect discernment of those preferences. The following example, which bears some similarity to Example 5 of Po0, shows how even slight uncertainty as to electorate preferences can cause RR2, RR3, RA2, and RA3 to have an advantage over RR1 and RA1 if there is a close contest between the two front-running candidates:

\section{Example 6.}

\begin{tabular}{lcccc}
\hline Group & $\mathrm{G}(\mathrm{ABC})$ & $\mathrm{G}(\mathrm{BAC})$ & $\mathrm{G}(\mathrm{CAB})$ & $\mathrm{G}(\mathrm{CBA})$ \\
\hline Number of voters & & & & \\
$\quad$ Actual & 40 & 45 & 9 & 6 \\
Believed to be & 40 & 45 & 11 & 4 \\
\hline RR1, RR2, or RR3 vote & $\mathrm{ACB} / \mathrm{ACB}$ & $\mathrm{BAC} / \mathrm{ABC}$ & $\mathrm{CAB} / \mathrm{ABC}$ & $\mathrm{CBA} / \mathrm{ABC}$ \\
\hline RA1 vote & $\mathrm{A} \mid \mathrm{CB}$ & $\mathrm{BA} \mid \mathrm{C}$ & $\mathrm{CA} \mid \mathrm{B}$ & $\mathrm{CB} \mid \mathrm{A}$ \\
\hline RA2 or RA3 vote & $\mathrm{A} \mid \mathrm{CB}$ & $\mathrm{B} \mid \mathrm{AC}$ & $\mathrm{CA} \mid \mathrm{B}$ & $\mathrm{CB} \mid \mathrm{A}$ \\
\hline
\end{tabular}

The preference order of the electorate is BAC with the actual profile but $\mathrm{ABC}$ with the presumed profile. For all six systems, all groups except $\mathrm{G}(\mathrm{ABC})$ follow their base strategies according to the presumed (not the actual) preference order, by using $\mathrm{ABC}$ for the electorate ranking on the RR ballots, or, on the RA ballots, by surmising A to be the Condorcet preference winner (and B the runner-up) when using Rule 1, 2, or 3 to mark approval votes.

Without the strategic voting by $\mathrm{G}(\mathrm{ABC})$, the actual Condorcet preference winner, $\mathrm{B}$, would straightforwardly win the race under any of the six systems. But the Condorcet vote cycle stemming from the manipulation by $\mathrm{G}(\mathrm{ABC})$ has to be resolved by tallying approval votes. The results are 94 for $\mathrm{A}$ and 51 for B (and 15 for C) under either RR1 or RA1, but are 51 for B and 49 for A under the other four systems. Thus, B still wins under those four but not under RR1 or RA1.

Of course, the $\mathrm{G}(\mathrm{BAC})$ voters could block the manipulation by voting BAC/BAC instead of $\mathrm{BAC} / \mathrm{ABC}$ under $\mathrm{RR} 1$ or $\mathrm{B} \mid \mathrm{AC}$ instead of $\mathrm{BA} \mid \mathrm{C}$ under RA1. They would have to violate base strategy to do so, though, whereas under the other four systems they face no similar dilemma. 
With the close race between $A$ and $B$, it does not seem sensible anyway for G(BAC) voters to award approval votes to $A$ as well as B, as under either RR1 or RA1. Incorrect or uncertain discernment of electorate preferences contributes to this conundrum. The plight of voters is actually greater under RR1: In the case of $m=3$, they control the approval votes (if they have to be tallied) directly and fully under RA1, but only indirectly under RR1.

\section{General Remarks}

This section is a collection of remarks that go beyond mathematical matters and deal with our framework and assumptions, practical and philosophical angles, and associated references.

Remark 10.1. Although goals (i) and (ii) stated at the start of the paper are not the only ones that one might want to examine, there is probably broad but not universal agreement that (i) and (ii) are both highly desirable. The selection of the Condorcet preference winner (i), including even the selection of the majority winner in the simple special case of a two-way race, may not maximize utility (in some form) in situations where preference intensities are distributed asymmetrically. Such situations, though, may be uncommon or at least not be easy to identify or remedy; and utility maximization is not the goal here anyway.

As for property (ii), it does not seem to receive high priority from (e.g.) advocates of the easily manipulable Borda procedure, or of plurality voting with its incentives to vote insincerely in some cases. But if a system does not reward misrepresentation and voters can vote their true preferences without fretting whether that is strategically unwise, they will face simpler tasks and will have greater confidence in the election process as well. Generally, the less manipulability, the better. Although one might prefer non-manipulability properties stronger than the Nash equilibria that are central to this paper, the nature of the problem limits what can be done.

For further discussion related to (i) and (ii), see (e.g.) Merrill [9] (especially Sections 1.2, 1.3, 3.1, and 5.1). For other viewpoints, see (e.g.) Saari [10] and Moulin [11] (Part IV). In addition, see Taylor [12] for an extensive treatment of manipulation in voting.

Remark 10.2. Instead of candidate, a broader term such as option could have been used throughout the paper. Options could refer not only to candidates in an election but also (e.g.) to rival legislative proposals. All of our results for candidates would hold also for other options.

Remark 10.3. In a system with an ordered agenda, voters vote on successive pairs of options. Other multi-stage systems also exist. We confine our attention, though, to voting systems that select a winner in a single stage.

With a single-stage system along with $m$ pre-specified options (or candidates), as in this paper, there is no room for undesirable agenda manipulation nor for strategic voting that could be used to counteract it, as covered in McKelvey [13]. Thus, although sophisticated voting may be beneficial in the agenda-manipulation framework by leading to improved outcomes, no such benefit would apply to our work here.

Remark 10.4. Although the Nash equilibria in our systems are relatively natural, understandable, and straightforward, there is, of course, no claim that any of them are unique. Other Nash equilibria would not generally have the same desirable properties as ours.

Remark 10.5. Computation for our systems may become prohibitive, when there is no Condorcet vote winner, if $m$ (the number of candidates) is too large. (But a big value of $n$ (number of voters) should 
cause little trouble.) Typical (e.g., single-digit) values of $m$ should be manageable. Moreover, computational burden can be greatly reduced when the shortcut described in Section 7 is applicable. Although the main calculations then involve just $m^{*}$ candidates (where $3 \leq m^{*} \leq m$ ), the value of $m^{*}$ cannot be known before the election.

In the worst case, $m^{*}=m$, one first applies Steps 1-3 (Section 3.2) to each of the $\left(\begin{array}{c}m \\ 3\end{array}\right)$ candidate triples. One then applies Steps $4\{4\}, 4\{5\}, \ldots, 4\{m\}$ successively to find the last-place candidate, L, in each of the $\left(\begin{array}{c}m \\ 4\end{array}\right)$ 4-tuples, $\left(\begin{array}{c}m \\ 5\end{array}\right)$ 5-tuples, and so on, where finding L may involve a candidate loop for some of these groups but will be simpler for others. The total number of groups (of varying size) is $\left(\begin{array}{c}m \\ 3\end{array}\right)+\left[\left(\begin{array}{c}m \\ 4\end{array}\right)+\left(\begin{array}{c}m \\ 5\end{array}\right)+\ldots+\left(\begin{array}{c}m \\ m\end{array}\right)\right]$, or $2^{m}-1-m-\left(\begin{array}{c}m \\ 2\end{array}\right)<2^{m}$, a number that would seem to be small enough to preclude prohibitive computational time so long as $m$ is below 10 or even slightly greater. Larger values of $m$ would probably be rare in practice anyway.

The task of writing an applicable computer program is a largely separate issue from avoidance of prohibitive computation. For small enough elections, hand calculations can be feasible, as one may gather from the examples in Section 4.

Remark 10.6. The concept of unilateral deviation or strategic voting by a homogeneous bloc, rather than a single voter, is not unique to this paper. Other works, such as Niou [14] and Brams and Sanver [6], as well as Po0, have also used it.

Remark 10.7. Note that we do not require true (or reported) preferences to be single-peaked. One would hardly expect voter preferences for $m>2$ candidates in most usual elections to be strictly single-peaked. (On the other hand, if voters are choosing not from $m$ candidates, but rather from (e.g.) numerical values along a continuum that represent money to be spent or taxed, then single-peaked preferences may be reasonable to assume. For such a case, methods far simpler than ours (e.g., asking voters to report just their first-preference value and then declaring the median to be the winner, as suggested long ago by Francis Galton) could be applied. See (e.g.) Black [15] (Chapter IV and p. 188) and Balinski and Laraki [16] (Section 5.2).)

Remark 10.8. The value of our systems is greater the lower the frequency of Condorcet preference cycles. Empirically, such cycles seem to be uncommon (see, e.g., Section 10 of Po0).

Remark 10.9. Positing knowledge of other voters' preferences, as this paper does, is not a novel concept and has a long tradition. The idea played a basic role (e.g.) in Farquharson's [17] (p. 38) classic development of "sophisticated voting"; in related schemes of McKelvey and Niemi [18]; and in the articles of Niemi and Frank [19], Eckel and Holt [20], and Felsenthal, Maoz, and Rapoport [21]. Both in those works and in the voting systems proposed here, each voter's knowledge of the preference profile of the electorate is the key to bringing about improvements in the voting process. More recent articles that assume voters' knowledge of other voters' preferences include Niou [14], Brams and Sanver [6], and Peress [7], as well as Po0. Any posited knowledge of electorate preferences is used only for determining voter strategies and, obviously, could not properly be used in any aggregation procedure.

One might be tempted to ask why an election is even needed at all if electorate preferences can already be discerned. Of course, not holding an election could hardly be legitimate.

Remark 10.10. How might voters obtain the needed information about the preference profile of the electorate? For various ideas and possibilities, see (e.g.) Section 9 of Po0. The information sources mentioned there include past election results, discussions in the media, and interactions with other voters. Especially, though, they include results from a certain type of public-opinion poll (Condorcet 
polling, covered in Potthoff [22]), which could apply in somewhat the same fashion as described in the work of McKelvey and Ordeshook [23] on rational-expectations equilibrium. More speculatively, they also include political stock markets.

Remark 10.11. To the extent that our systems work as intended, the discerned electorate preferences do not often play a role in the eventual aggregation, thus somewhat limiting their import. Paradoxically, their purpose is, in essence, to prevent them from being needed, or at least from needing to be used. Their function is to deter manipulation. If that deterrence succeeds, then manipulated vote cycles are not created, and only the voters' own reported preference rankings will generally play a role in the aggregation.

Remark 10.12. The voting systems of this paper (and of Po0) can be seen as falling under the wide umbrella of mechanism design (e.g., Moore [24]; Maskin [25]; Jackson [26]; Maskin and Sjöström [27]), sometimes referred to also as design of game forms or as reverse game theory. Mechanism design does cover voting systems but deals especially with economic problems. Typically, it does not involve preference groups of differing sizes, as in our work, nor use anything like our unusual ballots. Its results are often highly theoretical and abstract, and limited in their practicality (e.g., Moore [24] (pp. 209 ff.)) - unlike the systems of the present paper, which, though not as suited as those of Po0 to real-world elections, are nonetheless workable.

Remark 10.13. Ranking of $m$ candidates for either preferences or votes (Section 2 ) is more involved the larger $m$ is, although any ranked voting system becomes more burdensome for voters as $m$ increases. The points in Section 8 mitigate the complexities, though.

Although one could even question the assumption of transitivity of individual preferences (along the lines of Tversky [28], e.g.,), this issue would seem to be minor. For instance, the ABC exit poll among Anderson, Carter, and Reagan in the 1980 U.S. presidential election found individual intransitivity in only 52 respondents among 8146 (Blydenburgh [29] (p. 105)).

Remark 10.14. The RA ballot can be simply designed so as to require only a single mark beyond what an ordinary ranked ballot requests (cf. Po0, and the displays in the examples above).

Remark 10.15. Section 3.3 did not define the set $S$ for any case where no Condorcet preference winner is discerned (although for definiteness it could have done so). For some other cases, Section 3.3 could allow leeway for base strategies without affecting non-manipulability proofs but, for definiteness, it still defined S. But any indefiniteness concerning S does not prevent the use of any RR or RA system in an actual election, since the aggregation procedure is independent of $\mathrm{S}$ and is thus always well defined (though non-manipulability may fail in some cases).

Remark 10.16. Although Examples 3 and 4 in Section 5.2 prove that manipulation is indeed mathematically possible, the situations may be so complex that, practically speaking, voters may be unable to discover and carry out the successful manipulation strategies. Difficulty in understanding the role of the candidate triples and of the ascription of approval votes, along with uncertain discernment of electorate profiles, may deter manipulation efforts.

In general, the seriousness of whatever manipulability exists may largely depend not only on how easy or hard it is for voters to manipulate the system, but also on how often there are voter preference profiles where manipulation is even possible. These points may not be easy to judge.

Remark 10.17. Although we have pointed out some contrasts among the six systems of this paper, we have attempted no assessments to compare RR2 versus RR3 or RA2 versus RA3. 
Remark 10.18. Unfortunately, any voting system whose ballot asks for voters' candidate rankings and nothing more is doomed to be manipulable even in the rather extreme simple case where the domain of true-preference profiles is restricted to those that are single-peaked. This is shown by the following example, which resembles that of Blin and Satterthwaite [30] but is more general (it applies to any Condorcet completion method, not just Borda as in the earlier article) and is also like that of Penn, Patty, and Gailmard [31] (pp. 447-448) though the perspective differs:

Example 7. Suppose that 100 voters each report rankings of three candidates $X, Y$, and $Z$ with the result that there are 36 ballots marked XYZ (X first, Y second, Z third), 33 marked YZX, and 31 with ZXY. Thus, there is no Condorcet vote winner. Consider the following three voter preference profiles (each of which is consistent with single-peaked preferences):

\begin{tabular}{cccccccc}
\hline \multirow{2}{*}{ Profile } & \multicolumn{9}{c}{ Number of Voters Whose True Preference Order Is } & \multirow{2}{*}{\begin{tabular}{c} 
Condorcet \\
\cline { 2 - 6 }
\end{tabular}} & XYZ & XZY & YXZ & YZX & ZXY & ZYX & Preference Winner \\
\hline 1 & 36 & 0 & 33 & 0 & 31 & 0 & X \\
2 & 36 & 0 & 0 & 33 & 0 & 31 & Y \\
3 & 0 & 36 & 0 & 33 & 31 & 0 & Z \\
\hline
\end{tabular}

The observed ballot results could have arisen from Profile 1 if the 33 YXZ voters had voted YZX (and all others had voted their true preferences), from Profile 2 if ZYX voters had voted ZXY, or from Profile 3 if XZY voters had voted XYZ. From the ballots, there is no way to distinguish the three profiles with their three different Condorcet preference winners, even given that no more than one group sharing the same preference order deviated from voting its true preferences. Thus, no Condorcet completion method - including the many that have been proposed (e.g., Brams and Fishburn [4] (pp. 210-212); also Felsenthal and Machover [32])—would render the system non-manipulable, because the method could always select the "wrong" winner. That is, if it selected $Y(Z, X)$ and Profile $1(2,3)$ were the true profile, then the unilateral manipulation of YXZ (ZYX, XZY) voters in voting YZX (ZXY, XYZ) would be rewarded by the dethronement of $X(Y, Z)$ in favor of $Y(Z, X)$.

All is not lost, however. Manipulations like those in Example 7, and others that are far more involved, can be thwarted. Our work shows that non-manipulable systems do exist when the true preferences of the electorate show no cyclical majorities, or even when they show a Condorcet preference winner but may otherwise show cycle(s), if the ballots ask for certain markings in addition to the usual rankings.

Remark 10.19. The Impossibility Theorem of Arrow [33] and different results on ease of manipulation (e.g., Gibbard [34]; Satterthwaite [35]; Gärdenfors [36]) are not encouraging to those who try to find good voting systems. Nonetheless, as our work is intended to convey, the outlook is not as bleak as one might suppose, at least for situations where cycles in voter preferences are absent or of limited impact.

Remark 10.20. It may not be widely recognized that the Arrow results and manipulation results just mentioned do not apply if information is available that is not derivable from the true-preference profile. That is because the results follow from premises that assume availability of nothing other than the profile. They thus do not apply to approval voting nor, in fact, to any of the voting schemes covered here or in Po0. Of course, the unfavorable conclusions of those earlier works may well still hold under much broader premises (it would be highly optimistic to think otherwise), but different proofs would be 
required. Still, the lack of full applicability of the results may suggest that exploration of unusual voting systems can lead to unexpected benefits.

\section{Conclusions}

This paper, like an earlier one, Po0, develops single-winner voting systems that aim to elect a Condorcet preference winner while minimizing incentives for manipulation and insincere voting. A major objective is for voters to be able to vote sincerely with little need to consider whether doing so may hurt them or whether strategic voting might help them.

Both papers rely on discernment of electorate preferences, though less so in Po0. Po0 is less mathematical, but the systems of the present paper have stronger non-manipulability properties.

The Po0 systems are the simpler ones (and are even simpler than some currently used in certain public elections). Yet the systems of the present paper could be usable at least (e.g.) in a limited or sophisticated electorate, in a committee (whose members may know each other's views), in groups with some interest in voting theory, or in laboratory experiments, if not in a larger arena.

But leading contributions of the present paper, outside of its use of unusual ballots, lie in its distinctive mathematical aspects. These include its successive determination of last-place candidates in $j$-tuples, its use of candidate triples and of loops formed therefrom, and its non-manipulability proofs that use mathematical induction on $m$.

\section{Acknowledgments}

The author has benefited from helpful comments from Steven J. Brams, Samuel Merrill, Hannu Nurmi, and Mandar P. Oak, as well as from two referees. An earlier version of some of the material in this paper was presented at the annual meetings of the Public Choice Society in Nashville, Tennessee in March 2003. A related paper ("Super IRV: A cure for the myriad ills of instant-runoff voting?") was presented at the March 2011 Society meetings in San Antonio, Texas.

\section{Appendixes}

\section{A. Non-Manipulability of RR1, RR2, and RR3 When There Are No Preference Cycles}

This first appendix proves Proposition 2, which establishes that RR1, RR2, and RR3 are all non-manipulable for any number of candidates $(m)$ among whom there are no Condorcet preference cycles. The first step is to state and prove Theorem T1, whose proof uses Lemma L1 (proved earlier, in Section 5.1). Then Proposition 2 is proved, using Proposition 1 (also proved in Section 5.1) along with Theorem T1.

Theorem T1(m). Let $U_{m}$ be a set of $m(>2)$ candidates among whom the profile shows no Condorcet preference cycles. Let $A_{m}$ and $B_{m}$ then denote the candidates in $U_{m}$ who are the Condorcet preference winner and runner-up, respectively. Let $\mathrm{G}$ be any preference group. For any of the election systems RR1, RR2, or RR3, let S1 denote the set of base strategies, under which all voters simply mark their basic rankings according to their own true preferences, and mark their electorate rankings according to the Condorcet preference ranking for the electorate as a whole (i.e., $A_{m}$ first, $B_{m}$ second, and so on). If all 
preference groups other than G follow S1 but G can deviate from S1, then all of the following hold for each of RR1, RR2, and RR3:

$\mathrm{T} 1.1(m)$ : For any $\mathrm{G}$ that does not have $A_{m}$ as its first preference, the members of $\mathrm{G}$ can adopt no ballot strategy that will cause $A_{m}$ to place last in $U_{m}$.

T1.2( $m)$ : For any $\mathrm{G}$ with $B_{m}$ and $A_{m}$ as its first and second preferences, respectively, its members can adopt no ballot strategy that will prevent $A_{m}$ from placing above $B_{m}$ in $U_{m}$.

$\mathrm{T} 1.3(m)$ : For any $\mathrm{G}$ that has $A_{m}$ as its second preference and does not have $B_{m}$ as its first preference, the members of $\mathrm{G}$ can adopt no ballot strategy that will cause $B_{m}$ to place last in $U_{m}$.

Proof. The proof of Theorem T1 $(m)$ is by mathematical induction on $m$, and thus has two main steps. The first one is to prove T1(3). The $m=3$ candidates will be denoted by A $\left(=A_{3}\right), \mathrm{B}\left(=B_{3}\right)$, and $\mathrm{C}$ (Condorcet preference loser). There are, of course, $3 !=6$ preference groups $\mathrm{G}$.

Proof of T1.1(3). T1.1(3) applies to four of these six preference groups. Because G(BCA) and $\mathrm{G}(\mathrm{CBA})$ are already marking their basic rankings with A last, neither one can switch to a ballot strategy that will stop A from placing first. Because $\mathrm{G}(\mathrm{CAB})$ voters are already marking $\mathrm{C}$ first, they can do nothing to pull $\mathrm{C}$ ahead of either $\mathrm{A}$ or $\mathrm{B}$ in the two-way comparisons with each, so $\mathrm{C}$ must remain last. By L1.2 (see Section 5.1), G(BAC) voters cannot stop A from placing above B, regardless of whether the election system is RR1, RR2, or RR3.

Proof of T1.2(3). G(BAC) is the only applicable preference group. Again, the result follows from L1.2.

Proof of T1.3(3). The only applicable preference group is G(CAB). This group is unable to stop C from placing last, and so B cannot place last.

The other main step in the induction proof is to show that $\mathrm{T} 1(m+1)$ is true given that $\mathrm{T} 1(m)$ is true. For purposes of this step, T1.1 $(m+1)$ will be broken into three parts, as follows:

T1.1.1 $(m+1)$, Case 1: $\mathrm{G}$ has $A_{m+1}$ as third preference or lower.

T1.1.2 $(m+1)$, Case 2: G has $A_{m+1}$ as second preference, and $B_{m+1}$ is not first preference.

T1.1.3 $(m+1)$, Case 3: G has $A_{m+1}$ as second preference and $B_{m+1}$ as first preference.

T1.1.1 $(m+1)$, T1.1.2( $m+1)$, T1.1.3 $(m+1)$, T1.2 $(m+1)$, and T1.3 $(m+1)$, all given T1 $(m)$, will now each be proved in turn. The most intricate proof is the one for T1.1.3 $(m+1)$.

Proof of T1.1.1 $(m+1)$ given T1 $(m)$. G cannot have $A_{m+1}$ higher than second preference in any of the $m U_{m}$ sets that result from removing a candidate other than $A_{m+1}$ from $U_{m+1}$. Thus, by T1.1 $(m)$ with $A_{m+1}$ identified therein as $A_{m}$, it follows that $A_{m+1}$ cannot place last in any of these $m U_{m}$ sets, regardless of G's ballot strategy. It is therefore not possible for $A_{m+1}$ to place last in $U_{m+1}$, because to do so $A_{m+1}$ would have to place last in at least one of the $m U_{m}$ sets to which the candidate belongs.

Proof of T1.1.2( $m+1)$ given T1(m). In the $U_{m}$ set obtained by removing $A_{m+1}$ from $U_{m+1}$, no ballot strategy adopted by $\mathrm{G}$ can cause $B_{m+1}$ to place last, by virtue of T1.1 $(m)$ with $B_{m+1}$ identified therein as $A_{m}$. Similarly, in the $U_{m}$ set with $B_{m+1}$ removed from $U_{m+1}, \mathrm{G}$ cannot cause $A_{m+1}$ to place last, by virtue of T1.1 $(m)$ with $A_{m+1}$ identified therein as $A_{m}$. In any $U_{m}$ set obtained by removing any candidate other than $A_{m+1}, B_{m+1}$, or G's first preference from $U_{m+1}, A_{m+1}$ cannot place last, thanks to T1.1(m) with $A_{m+1}$ identified therein as $A_{m}$, and neither can $B_{m+1}$ place last, owing to T1.3( $\left.m\right)$ with $A_{m+1}$ and $B_{m+1}$ identified therein as $A_{m}$ and $B_{m}$, respectively. Thus, neither $A_{m+1}$ nor $B_{m+1}$ can place last in any of the $(m+1)$ possible $U_{m}$ sets except perhaps the one from which G's first-preference candidate is removed. This 
means that some candidate other than $A_{m+1}$ or $B_{m+1}$ has to place last in at least two of the $U_{m}$ sets, which precludes any possibility for either $A_{m+1}$ or $B_{m+1}$ to place last in $U_{m+1}$.

Note that the preceding argument not only proves T1.1.2 $(m+1)$ given $\mathrm{T} 1(m)$ but also proves $\mathrm{T} 1.3(m+1)$ given $\mathrm{T} 1(m)$.

Proof of T1.1.3( $\boldsymbol{m}+\mathbf{1})$ given T1 $(\boldsymbol{m})$. In any of the $(m-1)$ sets $U_{m}$ obtained by removing a candidate other than $A_{m+1}$ or $B_{m+1}$ from $U_{m+1}, A_{m+1}$ must place above $B_{m+1}$, no matter what ballot strategy $\mathrm{G}$ uses. This follows from T1.2( $m$ ) with $A_{m+1}$ and $B_{m+1}$ identified therein as $A_{m}$ and $B_{m}$, respectively. Thus, among the $(m+1)$ sets $U_{m}$ obtainable by dropping one candidate from $U_{m+1}, A_{m+1}$ can place last only in the $U_{m}$ set that results when $B_{m+1}$ is the candidate removed. In fact, $A_{m+1}$ must place last in that $U_{m}$ set if there is to be any possibility that $A_{m+1}$ will place last in $U_{m+1}$.

Moreover, if $A_{m+1}$ is to place last in $U_{m+1}$, the number of the $U_{m}$ sets in which $B_{m+1}$ places last cannot exceed 1 (since then $B_{m+1}$ would have more last places than $A_{m+1}$ ) and cannot be 0 (since then some other candidate would have more last places than $\left.A_{m+1}\right)$. Thus, $B_{m+1}$ must place last in exactly one of the $(m+1)$ sets $U_{m}$ to keep alive the possibility of $A_{m+1}$ placing last in $U_{m+1}$.

Next, because $A_{m+1}$ must place above $B_{m+1}$ in all sets $U_{m}$ that include both of these candidates, $A_{m+1}$ can place second from last in only one of these sets - the one in which $B_{m+1}$ places last. Arguing as before, one finds that $A_{m+1}$ must place next to last in this set, and also that $B_{m+1}$ must place second from last in exactly one set $U_{m}$ (if $A_{m+1}$ is to have any chance of placing last in $U_{m+1}$ ). Continued application of this argument leads to the conclusion that the $(m+1)$ sets $U_{m}$ have to consist of one set with $B_{m+1}$ first and $A_{m+1}$ omitted, one with $A_{m+1}$ first and $B_{m+1}$ second, one with $A_{m+1}$ second and $B_{m+1}$ third, ..., one with $A_{m+1}$ third from last and $B_{m+1}$ second from last, one with $A_{m+1}$ second from last and $B_{m+1}$ last, and one with $A_{m+1}$ last and $B_{m+1}$ omitted.

Thus, $A_{m+1}$ is a capping candidate, and $A_{m+1}$ caps $B_{m+1}$. Because $A_{m+1}$ is a capping candidate, $A_{m+1}$ cannot place last in $U_{m+1}$ unless all $(m+1)$ candidates are capping candidates. To complete the proof that $A_{m+1}$ cannot place last in $U_{m+1}$, it remains to deal with the case where every candidate is a capping candidate.

If all candidates are capping candidates, one examines the loop that they define, and the associated set of $(m+1)$ contiguous triples. The contiguous triple of which $B_{m+1}$ is the central member will also contain $A_{m+1}$. By L1.1, the number of approval votes for $B_{m+1}$ in this triple will be less than half the electorate (regardless of whether the election system is RR1, RR2, or RR3). Thus, $B_{m+1}$ is a lesser candidate. The contiguous triple of which $A_{m+1}$ is the central member will also contain $B_{m+1}$. By L1.1, the number of approval votes for $A_{m+1}$ in this triple will be more than half the electorate. Thus, $A_{m+1}$ is $n o t$ a lesser candidate. (Remember that approval votes are tallied for the central member of each contiguous triple even if the triple is noncyclical.)

Finally, because there is at least one lesser candidate $\left(B_{m+1}\right)$, and because $A_{m+1}$ is not a lesser candidate, the last-place candidate in $U_{m+1}$ will be chosen from candidates who do not include $A_{m+1}$. This eliminates the only remaining avenue by which $A_{m+1}$ could place last in $U_{m+1}$.

Proof of T1.2( $m+1)$ given T1 $(m)$. By T1.1 $(m+1)$ (which was just proved), $A m+1$ cannot place last in $U_{m+1}$, regardless of what ballot strategy $\mathrm{G}$ uses. If $B_{m+1}$ places last in $U_{m+1}$, the conclusion of T1.2 $(m+1)$ is immediate. Thus, one needs to consider only the case where some candidate other than $A_{m+1}$ or $B_{m+1}$ places last in $U_{m+1}$. The proof of T1.2(m+1) is completed by applying T1.2( $\left.m\right)$, with $A_{m+1}$ 
and $B_{m+1}$ identified therein as $A_{m}$ and $B_{m}$, respectively, to the $U_{m}$ set that is obtained by removing the last-place candidate from $U_{m+1}$.

Proof of T1.3( $\boldsymbol{m}+\mathbf{1})$ given T1 $(\boldsymbol{m})$. See proof of T1.1.2 $(m+1)$ given T1 $(m)$, above.

Proposition 2. Let there be $m$ candidates with the same conditions and notation as in Theorem T1. (Thus, the domain for admissible true-preference profiles is F0.) Then each of the systems RR1, RR2, and RR3 is non-manipulable. That is, no preference group $\mathrm{G}$, by deviating from $\mathrm{S} 1$ when no one else does, can adopt a ballot strategy that will dethrone $A_{m}$ (the Condorcet preference winner) as the election winner in favor of a candidate whom $\mathrm{G}$ prefers to $A m$.

Proof. G cannot gain by deviating from $\mathrm{S} 1$ if $\mathrm{G}$ has $A m$ as its first preference. With this case disregarded, T1.1 $(\mathrm{m})$ implies that $\mathrm{G}$ can adopt no ballot strategy that will cause $A_{m}$ to place last in $U_{m}$. Therefore some other candidate will have to place at the bottom of $U_{m}$. Let $U_{m-1}$ denote the set obtained by removing that candidate from $U_{m}$. If $A_{m}$ is G's top preference within $U_{m-1}$, then G can achieve nothing better than the election of $A_{m}$. If not, then apply T1.1 $(m-1)$ to $U_{m-1}$, with $A_{m}$ identified therein as $A_{m-1}$, to conclude that $\mathrm{G}$ can do nothing to cause $A_{m}$ to place last in $U_{m-1}$. Let $U_{m-2}$ denote the set obtained by removing from $U_{m-1}$ the candidate who does place last in $U_{m-1}$. If $A_{m}$ is G's first preference in $U_{m-2}$, then, as before, the conclusion is proved. If not, then apply T1.1 $(m-2)$. Continue in this way until either the conclusion is proved because $A_{m}$ is G's top preference among remaining (non-excluded) candidates or else a candidate (other than $A_{m}$ ) is removed from $U_{4}$ to produce $U_{3}$. In the latter case, apply Proposition 1 to show that $\mathrm{G}$ can do nothing in $U_{3}$ to dethrone $A_{m}$ in favor of another candidate in $U_{3}$ whom $\mathrm{G}$ prefers to $A_{m}$.

\section{B. Non-Manipulability of RRI and RAI When There Is a Condorcet Preference Winner}

This appendix proves Proposition 3, which establishes that RR1 and RA1 are both non-manipulable for any number of candidates $(m)$ among whom there is a Condorcet preference winner but not necessarily an absence of Condorcet preference cycles otherwise. The development begins with Lemma L2, which is analogous to Lemma L1 (see Section 5.1). Then comes Lemma L3, whose proof uses Lemma L2; L3 is analogous to Proposition 1 (also in Section 5.1). Next is Theorem T2, which is analogous to Theorem T1 (Appendix A) and whose proof uses L2. Finally, Proposition 3 is analogous to Proposition 2 (Appendix A) and is proved with the help of L3 and T2.

Lemma L2. Let there be a set of three candidates A, B, and C who are, respectively, the Condorcet preference winner, runner-up, and loser. Under the system RR1, let S1* denote the set of ballot strategies under which all voters mark their basic rankings according to their own true preferences, and mark their electorate rankings either as A first, B second, and C third or as A first, C second, and B third. Under RA1, let S2* denote the set of ballot strategies under which all voters mark their basic rankings according to their own true preferences, and mark their approval votes in accord with Rule 1 (that is, their approval votes go only to A if $\mathrm{A}$ is their first preference, or to their first two preferences otherwise). Let $\mathrm{G}^{*}$ refer to either $\mathrm{G}(\mathrm{BAC})$ or $\mathrm{G}(\mathrm{CAB})$ (but not to both simultaneously), and let $\mathrm{G}^{* *}$ refer to the other one. For either RR1 or RA1, suppose that all preference groups other than $\mathrm{G}^{*}$ follow $\mathrm{S} 1 *$ or $\mathrm{S} 2 *$ (respectively) but that $\mathrm{G}^{*}$ can deviate from $\mathrm{S} 1 *$ or $\mathrm{S} 2 *$. Then both of the following will hold no matter what ballot strategy $\mathrm{G}^{*}$ adopts:

L2.1: If approval votes are tallied using the applicable rules (whether or not the three candidates show cyclical vote majorities that trigger such a tally), then A will receive approval votes from more than half 
the electorate and $B$ from less than half if $\mathrm{G}^{*}$ is $\mathrm{G}(\mathrm{BAC})$, and A will receive approval votes from more than half the voters and $C$ from less than half if $G^{*}$ is $G(C A B)$.

L2.2: Within the set of three candidates, $A$ will place above $B$ if $G^{*}$ is $G(B A C)$ and above $C$ if $G^{*}$ is $\mathrm{G}(\mathrm{CAB})$.

Proof of L2.1. Under either RR1 or RA1 (and under S1* or S2*, respectively), G(ABC) and G(ACB) provide approval votes only to $\mathrm{A}$, and $\mathrm{G}^{* *}$ gives approval votes to its top two preferences. Thus, $\mathrm{G}(\mathrm{ABC}), \mathrm{G}(\mathrm{ACB})$, and $\mathrm{G}^{* *}$ provide approval votes to $\mathrm{A}$ but not to $\mathrm{G}^{*}$ 's most preferred candidate. Those are the three groups that prefer $A$ to $G^{*}$ 's top preference (B or C) and thus constitute more than half the electorate. L2.1 then follows.

Proof of L2.2. The argument is like that in the proof of L1.2.

Lemma L3. Let there be $m=3$ candidates with the same conditions and notation as in the first three sentences of Lemma L2. Then each of the systems RR1 and RA1 is non-manipulable. That is, no preference group $\mathrm{G}$, by deviating from $\mathrm{S} 1 *$ or $\mathrm{S} 2 *$ when no one else does, can adopt a ballot strategy that will dethrone $\mathrm{A}$ (the Condorcet preference winner) as the election winner in favor of a candidate whom G prefers to A.

Proof for RA1. For RA1 the proof is already covered by Proposition 1, because S2* in Lemma L3 and Lemma L2 is the same as S2 in Proposition 1 and Lemma L1.

Proof for RR1. Because S1* here and S1 earlier are not likewise the same, for RR1 the proof of Proposition 1 has to be modified slightly. Again the proof considers each of the six preference groups separately. The argument is the same as before for the five preference groups other than G(BAC). Then, from $L 2.2$ (where $G^{*}$ is $\mathrm{G}(\mathrm{BAC})$ ) it follows that $\mathrm{G}(\mathrm{BAC})$ cannot stop A from placing above B, and thus can bring about nothing better than the election of $\mathrm{A}$.

Theorem T2(m). Let $U_{m}$ be a set of $m(>2)$ candidates that includes a Condorcet preference winner, to be denoted by $A_{m}$, but otherwise may have Condorcet preference cycle(s). Let $\mathrm{G}$ be any preference group. For the election system RR1, let $\mathrm{S} 1 *$ denote any set of ballot strategies under which all voters simply mark their basic rankings according to their own true preferences, and mark their electorate rankings with $A_{m}$ ranked first but with the other candidates in any order. For RA1, let S2* denote the set of ballot strategies under which all voters mark their basic rankings according to their own true preferences, and mark their approval votes in accord with Rule 1 (which means that their approval votes go to $\mathrm{A}$ and to any candidate preferred to A unless A is their lowest preference, in which case all candidates except A receive their approval votes). For either RR1 or RA1, if all preference groups other than $\mathrm{G}$ follow $\mathrm{S} 1$ * or S2* (respectively) but $\mathrm{G}$ can deviate from $\mathrm{S} 1$ * or S2*, then the following will hold:

T2.1 $(m)$ : For any $\mathrm{G}$ that does not have $A_{m}$ as its first preference, the members of $\mathrm{G}$ can adopt no ballot strategy that will cause $A_{m}$ to place last in $U_{m}$.

T2.2 $(m)$ : For any $\mathrm{G}$ with $A_{m}$ as its second preference and some other candidate $W_{m}$ as its first preference, its members can adopt no ballot strategy that will prevent $A_{m}$ from placing above $W_{m}$ in $U_{m}$.

Proof. The proof of Theorem T2 $(m)$ is much like that of T1 $(m)$. It uses mathematical induction again and starts by proving T2(3). Then it proves T2 $(m+1)$ given T2 $(m)$. For RR1 and RA1 the arguments are essentially the same.

Proof of T2.1(3). With $m=3$ (though not when $m>3$ ), there can be no Condorcet preference cycle, because it is given that $A_{3}$ (hereafter $\mathrm{A}$ ) is the Condorcet preference winner. The premises of Lemma L2 are thus satisfied. Then T2.1(3) follows from L2.2 if G is G(BAC) or G(CAB), where B and C denote 
(respectively) the Condorcet preference runner-up and loser within the set of three candidates. For the other two applicable preference groups, $\mathrm{G}(\mathrm{BCA})$ and $\mathrm{G}(\mathrm{CBA})$, the proof of $\mathrm{T} 2.1(3)$ is the same as that for $\mathrm{T} 1.1(3)$.

Proof of T2.2(3). G(BAC) and $\mathrm{G}(\mathrm{CAB})$ are the only applicable preference groups. T2.2(3) follows from L2.2.

For purposes of proving $\mathrm{T} 2(m+1)$ given $\mathrm{T} 2(m), \mathrm{T} 2.1(m+1)$ will be broken into two parts, as follows:

T2.1.1 $(m+1)$, Case 1: $\mathrm{G}$ has $A m+1$ as third preference or lower.

T2.1.2 $(m+1)$, Case 2: $\mathrm{G}$ has $A_{m+1}$ as second preference.

T2.1.1 $(m+1)$, T2.1.2( $m+1)$, and T2.2 $(m+1)$, all given T2 $(m)$, will each be proved in turn.

Proof of T2.1.1 $(m+1)$ given T2 $(m)$. The proof follows the same wording as in the proof of $\mathrm{T} 1.1 .1(m+1)$ given $\mathrm{T} 1(m)$ (see Appendix A), except that "T1.1 $(m)$ " therein is to be replaced by "T2.1(m)."

Proof of T2.1.2( $\boldsymbol{m}+\mathbf{1})$ given T2(m). Let $W_{m+1}$ denote the first-preference candidate of $\mathrm{G}$ within $U_{m+1}$. Then the proof follows the same wording as the proof of T1.1.3( $\left.m+1\right)$ given T1 $(m)$, except that " $B_{m+1}$ " therein is to be replaced (wherever " $B_{m+1}$ " appears) by " $W_{m+1}$," " $B_{m}$ " is to be replaced by " $W_{m}$," "T1.2 $(m)$ " by "T2.2 $(m)$," and "L1.1" by "L2.1." In addition, the reference to "RR1, RR2, or RR3" is to be changed to "RR1 or RA1."

Proof of T2.2( $m+1)$ given T2(m). The proof follows the same wording as the proof of T1.2( $m+1)$ given T1 $(m)$, except that " $B_{m+1}$ " therein is to be replaced by " $W_{m+1}$, " $B_{m}$ " by " $W_{m}$," "T1.1 $(m+1)$ " by “T2.1 $(m+1)$," "T1.2( $m+1)$ " by "T2.2( $m+1)$," and "T1.2 $(m)$ " by "T2.2 $(m)$."

Proposition 3. Let there be $m$ candidates with the same conditions and notation as in Theorem T2. (In particular, the domain for admissible true-preference profiles is F1.) Then each of the systems RR1 and RA1 is non-manipulable. That is, no preference group G, by deviating from S1* (for RR1) or S2* (for RA1) when no one else does, can adopt a ballot strategy that will dethrone $A m$ (the Condorcet preference winner) as the election winner in favor of a candidate whom G prefers to $A_{m}$.

Proof. For either RR1 or RA1, the proof has the same wording as the proof of Proposition 2, except that "S1" therein is to be changed to "S1* or $\mathrm{S} 2 *$," "T $1.1(m)$ " to "T2.1 $(m)$, , "T $1.1(m-1)$ " to "T2.1(m-1)," "T1.1 $(m-2)$ " to "T2.1 $(m-2)$," and "Proposition 1" to "Lemma L3."

\section{Validity of Restricting Condorcet Completion Method to the Top Vote Cycle}

Proposition 4 establishes a way to simplify aggregation (see Section 7).

Proposition 4. Consider any of the four aggregation procedures (for RR1, RR2, or RR3, or for the RA ballot). Suppose that there is no Condorcet vote winner and that the top vote cycle consists of $m^{*}$ $\left(3 \leq m^{*} \leq m\right)$ of the $m$ candidates. Call any candidate in the top vote cycle a B candidate, and call any other candidate a $\mathrm{W}$ candidate. Then both of the following hold:

P4.1(j): When the Condorcet completion method associated with the aggregation procedure is applied to all $m$ candidates, no $\mathrm{W}$ candidate will place above any B candidate in any subset consisting of $j(3 \leq j \leq m)$ of the $m$ candidates.

P4.2: The order in which the $m^{*} \mathrm{~B}$ candidates finally place will be the same whether the Condorcet completion method is applied to all $m$ candidates or just to these $m^{*}$ candidates. 
Proof. The same proof of Proposition 4 applies to all four of the aggregation procedures.

Proof of P4.1(j). The proof is by mathematical induction on $j$ (for any fixed $m$ ). We first prove P4.1(3), and then prove P4.1 $(j+1)$ given P4.1(j).

Proof of P4.1(3). The result is trivially true if the three candidates are either all B's or all W's. If there are two B's and one W or vice versa, then the triple must be noncyclical and no $\mathrm{W}$ can place above a B; this is because no $\mathrm{W}$ is rated above a $\mathrm{B}$ in the basic rankings.

Proof of P4.1 $(j+1)$ given P4.1(j). Let $U_{j+1}$ denote any set of $(j+1)$ of the $m$ candidates. If there are no W's in $U_{j+1}$, then P4.1 $(j+1)$ is trivially true. If $U_{j+1}$ has exactly one $\mathrm{W}$, then, by P4.1 $(j)$, that W places at the bottom of all but one of the $(j+1)$ subsets of $U_{j+1}$ that are of size $j$, and thus places at the bottom of $U_{j+1}$. If $U_{j+1}$ has two or more W's, then P4.1(j) implies that all $(j+1)$ of the subsets must have a W placing at the bottom. Thus, a $\mathrm{W}$ must place at the bottom of $U_{j+1}$. Because the remaining candidates are in the same order as in the subset that contains them all, no $\mathrm{W}$ can place above a B in that subset (again by $\mathrm{P} 4.1(j))$ nor (therefore) in $U_{j+1}$ itself.

Proof of P4.2. By P4.1 $(m)$, the $m^{*}$ B candidates will place at the top of the $m$ candidates when the Condorcet completion method is applied to all $m$ candidates. Moreover, by virtue of the aggregation procedure, the ordering of these $m^{*}$ candidates at the top will be the same as in the subset that consists of them alone. This is, of course, the same ordering that is obtained upon applying the Condorcet completion method to just those $m^{*}$ candidates.

\section{Conflicts of Interest}

The author declares no conflict of interest.

\section{References}

1. Potthoff, R.F. Simple manipulation-resistant voting systems designed to elect Condorcet candidates and suitable for large-scale public elections. Soc. Choice Welf. 2013, 40, 101-122.

2. Brams, S.J.; Fishburn, P.C. Approval Voting, 2nd ed.; Springer: New York, NY, USA, 2007.

3. Nurmi, H. Comparing Voting Systems; Reidel: Dordrecht, The Netherlands, 1987.

4. Brams, S.J.; Fishburn, P.C. Voting procedures. In Handbook of Social Choice and Welfare; Arrow, K.J., Sen, A.K., Suzumura, K., Eds.; Elsevier: Amsterdam, The Netherlands, 2002; Volume 1, pp. 173-236.

5. Tideman, N. Collective Decisions and Voting: The Potential for Public Choice; Ashgate: Aldershot, England, UK, 2006.

6. Brams, S.J.; Sanver, M.R. Critical strategies under approval voting: Who gets ruled in and ruled out. Elect. Stud. 2006, 25, 287-305.

7. Peress, M. Selecting the Condorcet winner: Single-stage versus multi-stage voting rules. Public Choice 2008, 137, 207-220.

8. Pritchard, G.; Wilson, M.C. Exact results on manipulability of positional voting rules. Soc. Choice Welf. 2007, 29, 487-513.

9. Merrill, S. Making Multicandidate Elections More Democratic; Princeton University Press: Princeton, NJ, USA, 1988.

10. Saari, D.G. Geometry of Voting; Springer-Verlag: Berlin, Germany, 1994. 
11. Moulin, H. Axioms of Cooperative Decision Making; Cambridge University Press: Cambridge, England, UK, 1988.

12. Taylor, A.D. Social Choice and the Mathematics of Manipulation; Cambridge University Press: Cambridge, England, UK, 2005.

13. McKelvey, R.D. Intransitivities in multidimensional voting models and some implications for agenda control. J. Econ. Theory 1976, 12, 472-482.

14. Niou, E.M.S. Strategic voting under plurality and runoff rules. J. Theor. Politics 2001, 13, 209-227.

15. Black, D. The Theory of Committees and Elections; Cambridge University Press: Cambridge, England, UK, 1958.

16. Balinski, M.; Laraki, R. Majority Judgment: Measuring, Ranking, and Electing; MIT Press: Cambridge, MA, USA, 2010.

17. Farquharson, R. Theory of Voting; Yale University Press: New Haven, CT, USA, 1969.

18. McKelvey, R.D.; Niemi, R.G. A multistage game representation of sophisticated voting for binary procedures. J. Econ. Theory 1978, 18, 1-22.

19. Niemi, R.G.; Frank, A.Q. Sophisticated voting under the plurality procedure: A test of a new definition. Theor. Decis. 1985, 19, 151-162.

20. Eckel, C.; Holt, C.A. Strategic voting in agenda-controlled committee experiments. Am. Econ. Rev. 1989, 79, 763-773.

21. Felsenthal, D.S.; Maoz, Z.; Rapoport, A. The Condorcet-efficiency of sophisticated voting under the plurality and approval procedures. Behav. Sci. 1990, 35, 24-33.

22. Potthoff, R.F. Condorcet polling. Public Choice 2011, 148, 67-86.

23. McKelvey, R.; Ordeshook, P.C. Rational expectations in elections: Some experimental results based on a multidimensional model. Public Choice 1984, 44, 61-102.

24. Moore, J. Implementation, contracts, and renegotiation in environments with complete information. In Advances in Economic Theory, Sixth World Congress; Laffont, J.-J., Ed.; Cambridge University Press: Cambridge, England, UK, 1992; Volume 1, pp. 182-282.

25. Maskin, E. Nash equilibrium and welfare optimality. Rev. Econ. Stud. 1999, 66, 23-38.

26. Jackson, M.O. A crash course in implementation theory. Soc. Choice Welf. 2001, 18, 655-708.

27. Maskin, E.; Sjöström, T. Implementation theory. In Handbook of Social Choice and Welfare; Arrow, K.J., Sen, A.K., Suzumura, K., Eds.; Elsevier: Amsterdam, The Netherlands, 2002; Volume 1, pp. 237-288.

28. Tversky, A. Intransitivity of preferences. Psychol. Rev. 1969, 76, 31-48.

29. Blydenburgh, J.C. Sophisticated voting in the 1980 presidential election. Polit. Behav. 1988, 10, 103-116.

30. Blin, J.M.; Satterthwaite, M.A. Strategy-proofness and single-peakedness. Public Choice 1976, 26, 51-58.

31. Penn, E.M.; Patty, J.W.; Gailmard, S. Manipulation and single-peakedness: A general result. Am. J. Polit. Sci. 2011, 55, 436-449.

32. Felsenthal, D.S.; Machover, M. After two centuries, should Condorcet's voting procedure be implemented? Behav. Sci. 1992, 37, 250-274.

33. Arrow, K.J. Social Choice and Individual Values, 2nd ed.; Wiley: New York, NY, USA, 1963. 
34. Gibbard, A. Manipulation of voting schemes: A general result. Econometrica 1973, 41, 587-601.

35. Satterthwaite, M.A. Strategy-proofness and Arrow's conditions: Existence and correspondence theorems for voting procedures and social welfare functions. J. Econ. Theory 1975, 10, 187-217.

36. Gärdenfors, P. Manipulation of social choice functions. J. Econ. Theory 1976, 13, 217-228.

(C) 2014 by the author; licensee MDPI, Basel, Switzerland. This article is an open access article distributed under the terms and conditions of the Creative Commons Attribution license (http://creativecommons.org/licenses/by/4.0/). 